\title{
THE MICROJANSKY RADIO GALAXY POPULATION ${ }^{1}$
}

\author{
A. J. BArger, ${ }^{2,3,4}$ L. L. Cowie, ${ }^{4}$ W.-H. WANG ${ }^{4}$ \\ Accepted by The Astrophysical Journal for January 1, 2007
}

\begin{abstract}
We use highly spectroscopically complete observations of the radio sources from the VLA $1.4 \mathrm{GHz}$ survey of the Hubble Deep Field-North region to study the faint radio galaxy population and its evolution. The fraction of radio sources that can be optically spectroscopically identified is fairly independent of radio flux, with about $60-80 \%$ identified at all fluxes. We spectrally classify the sources into four spectral types: absorbers, star formers, Seyfert galaxies, and broad-line AGNs, and we analyze their properties by type. We supplement the spectroscopic redshifts with photometric redshifts measured from the restframe ultraviolet to mid-infrared spectral energy distributions. Using deep X-ray observations of the field, we do not confirm the existence of an X-ray-radio correlation for star-forming galaxies. We also do not observe any correlations between $1.4 \mathrm{GHz}$ flux and $R$ magnitude or redshift. We find that the radio powers of the host galaxies rise dramatically with increasing redshift, while the optical properties of the host galaxies show at most small changes. Assuming that the locally determined far-infrared (FIR)-radio correlation holds at high redshifts, we estimate total FIR luminosities for the radio sources. We note that the FIR luminosity estimates for any radio-loud AGNs, which we conservatively do not try to remove from the sample, will be overestimates. Considering only the radio sources with quasar-like bolometric luminosities, we find a maximum ratio of candidate highly-obscured AGNs to X-ray-luminous $\left(L_{0.5-2} \mathrm{keV}\right.$ or $\left.L_{2-8 \mathrm{keV}} \geq 10^{42} \mathrm{ergs} \mathrm{s}^{-1}\right)$ sources of about 1.9. Finally, we use source-stacking analyses to measure the X-ray surface brightnesses of various X-ray and radio populations. We find the contributions to the 4-8 keV light from our candidate highly-obscured AGNs to be very small, and hence these sources are unable to account for the light that has been suggested may be missing at these energies.
\end{abstract}

Subject headings: cosmology: observations — galaxies: active — galaxies: distances and redshifts — galaxies: evolution - galaxies: formation

\section{INTRODUCTION}

In the local universe, Seyfert 2 galaxies outnumber Seyfert 1 galaxies by 4 to 1 (Maiolino \& Rieke 1995), and more than half of Seyfert 2 nuclei are Comptonthick with obscuring column densities $N_{H}>10^{24} \mathrm{~cm}^{-2}$ (Maiolino et al. 1998; Risalti et al. 1999; Guainazzi et al. 2005; Comastri 2004). In the high-redshift universe, Xray background population synthesis models (refined after the discovery of a lower redshift distribution than expected for the hard X-ray sources; e.g., Barger et al. 2002, 2003; Szokoly et al. 2004) similarly predict a population of highly-obscured active galactic nuclei (AGNs) that are missed in the deep Chandra and XMM-Newton hard X-ray surveys (e.g., Franceschini et al. 2002; Gandhi \& Fabian 2003; Ueda et al. 2003; Comastri 2004; Gilli 2004; Fabian \& Worsley 2004; Treister \& Urry 2005; Ballantyne et al. 2006). Such theoretical predictions appear to be receiving some observational confirmation in the work of Worsley et al. (2005). Using a source-stacking analysis on the Chandra Deep Field-North (CDF-N) and Chandra Deep FieldSouth (CDF-S) fields, Worsley et al. (2005) found that the resolved fraction in the $6-8 \mathrm{keV}$ band was only about $60 \%$. (Worsley et al. 2004 did a similar analysis on the XMMNewton Lockman Hole field and found a $50 \%$ resolved frac- tion above $8 \mathrm{keV}$.) According to these authors, the missing $\mathrm{X}$-ray background (XRB) component has a spectral shape consistent with a population of highly-obscured AGNs at redshifts $z \sim 0.5-1.5$ having obscuring column densities of $N_{H} \sim 10^{23}-10^{24} \mathrm{~cm}^{-2}$.

Of course, to derive these results, Worsley et al. (2005) had to choose which estimate of the $2-8 \mathrm{keV}$ extragalactic XRB spectrum to use, and there is a great deal of uncertainty in these measurements (see Figure 15 of Hickox \& Markevitch 2006 for a summary; Worsley et al. 2005 used the XMM-Newton measurement made by Deluca \& Molendi 2004, but Revnivtsev et al. 2005 found that the intensity of the XRB measured by focusing telescopes is higher than that measured by collimated experiments by about $10-15 \%$, possibly due to the extreme complexity of measuring effective solid angles of focusing telescopes). Indeed, Barger et al. (2002) found that the issue of whether there is a need for a substantial population of as-yet undetected highly-obscured AGNs depends critically on how the low-energy and high-energy XRB measurements tie together.

Regardless, the combination of the theoretical modelling predictions for a population of highly-obscured AGNs missing from the deep X-ray surveys and the possible ob-

\footnotetext{
${ }^{1}$ Based in part on data obtained at the W. M. Keck Observatory, which is operated as a scientific partnership among the the California Institute of Technology, the University of California, and NASA and was made possible by the generous financial support of the W. M. Keck Foundation.

${ }^{2}$ Department of Astronomy, University of Wisconsin-Madison, 475 North Charter Street, Madison, WI 53706

${ }^{3}$ Department of Physics and Astronomy, University of Hawaii, 2505 Correa Road, Honolulu, HI 96822

${ }^{4}$ Institute for Astronomy, University of Hawaii, 2680 Woodlawn Drive, Honolulu, HI 96822
} 
servational evidence for a substantial unresolved fraction of the XRB has inspired observers to try to quantify the fraction of obscured AGNs that may be being missed. Deep mid-infrared (MIR) and radio images are an obvious avenue for searching for highly-obscured AGNs, since extinction in the MIR and radio is small. Various approaches using MIR or X-ray data (e.g., Alonso-Herrero et al. 2006; Polletta et al. 2006), the combination of MIR and radio data (e.g., Martínez-Sansigre et al. 2005), or the combination of MIR, radio, and X-ray data (e.g., Donley et al. 2005) have been adopted, and a candidate population of highly-obscured AGNs has been identified. However, the various selection effects in these approaches mean that a reliable upper limit on the number of highly-obscured AGNs that are undetected even in the $2-8 \mathrm{keV}$ band has not yet been obtained.

In this paper, we take an alternative approach to look for highly-obscured AGNs at high redshifts using a pure microJansky radio survey selection. Ultradeep radio surveys are very useful for tracing the evolution of dust-obscured galaxies and AGNs. In contrast to optical surveys, which may omit dusty sources, and submillimeter surveys, which currently have low spatial resolution and are limited to small areas for any significant depth, the $1.4 \mathrm{GHz}$ surveys do not suffer from extinction, provide subarcsecond positional accuracy, and cover large areas.

Although the Jansky and milliJansky radio source populations are dominated by powerful AGNs, at the microJansky level, the radio source population is increasingly dominated by star-forming galaxies, which produce non-thermal radio continuum at $1.4 \mathrm{GHz}$ through synchrotron emission from supernova remnants. In local starforming galaxies and radio-quiet AGNs, it is well-known that the radio power is tightly correlated with the farinfrared (FIR) luminosity (e.g., Helou et al. 1985; Condon et al. 1991; Condon 1992), probably as a result of both being linearly related to the massive star formation rate. If we assume that this correlation continues to hold at high redshifts (note that there is some observational support that the correlation does extend to $z \geq 1$ from the work of Appleton et al. 2004, though the number of galaxies at the higher redshifts is relatively low), then we can use this relation to estimate the total FIR luminosity of a galaxy from its radio power alone. Indeed, even with the advent of the Spitzer Space Telescope, this is still the most robust way to estimate a galaxy's total FIR luminosity, due to the limited MIPS sensitivities at 70 and $160 \mu \mathrm{m}$ and the uncertainties in the conversion from $24 \mu \mathrm{m}$ luminosity to total FIR luminosity. Although estimating total FIR luminosities from $24 \mu \mathrm{m}$ data has been advocated (e.g., Appleton et al. 2004; Marcillac et al. 2006), the conversion depends on the assumed shape of the spectral energy distribution (SED), which is expected to change as a function of dust temperature and metallicity.

Using their Spitzer Infrared Nearby Galaxies Survey (SINGS) data, Dale et al. (2005) found a total range in rest-frame $8 \mu \mathrm{m}$ (which corresponds to observed-frame $24 \mu \mathrm{m}$ at $z=2$ ) to total FIR luminosity ratios of more than a factor of 20. When they excluded the metal-poor dwarfs, the total range dropped to a factor of 10, which they argued should be taken as a minimum systematic uncertainty for total FIR luminosities inferred from restframe $8 \mu \mathrm{m}$ fluxes alone. They cautioned that if the metal abundance of the population cannot be inferred a priori, then the uncertainties may be considerably higher. Likewise, they found that the rest-frame $24 \mu \mathrm{m}$ to total FIR ratio spans a factor of 5 in the SINGS sample.

In addition to our assumption that the FIR-radio correlation continues to hold at high redshifts, the other possible concern with our procedure of estimating the total FIR luminosities of the radio sources using the FIR-radio correlation is the presence of radio-loud AGNs. For these sources, the radio continuum will be in excess of the level expected from star formation, and we will overestimate their total FIR luminosities. Fortunately, however, since we are only interested in obtaining upper limits on the number of highly-obscured AGNs, this is not a major concern for our present analysis, and we have conservatively not made any effort to remove such sources from our sample.

Finally, we note that even in the case of the radio-quiet AGNs, we are not able to separate out the AGN contribution to the FIR luminosity from the star-forming contribution. However, since local radio-quiet AGNs are empirically observed to follow the FIR-radio correlation, we have an upper limit on the AGN FIR emission, which is sufficient for our analysis.

In this paper, we use the ultradeep $1.4 \mathrm{GHz}$ observations of the Hubble Deep Field-North (HDF-N; Richards 2000), for which we have obtained very complete spectroscopic identifications, to study the properties of the radio sample. The HDF-N has a wealth of existing multiwavelength data, including the HST ACS Treasury Great Observatories Origins Deep Survey-North data (GOODS-N; Giavalisco et al. 2004) and the Spitzer Space Telescope Legacy GOODS-N data (P.I. M. Dickinson). In addition, we have recently obtained deep wide-area $J$ - and $H$-band observations using the University of Hawaii $2.2 \mathrm{~m}$ telescope (Trouille et al. 2006, in preparation), which enable us to assign robust photometric redshifts to the spectroscopically unidentified sources.

The HDF-N is also the site of the deepest X-ray image of the sky, the $2 \mathrm{Ms}$ CDF-N. We use these data to examine the issue of whether there exists an X-ray-radio correlation for star-forming galaxies. In addition, we use these data to remove the X-ray-luminous $\left(L_{0.5-2} \mathrm{keV}\right.$ or $L_{2-8} \mathrm{keV} \geq 10^{42} \mathrm{ergs} \mathrm{s}^{-1}$ ) sources from our radio-identified candidate highly-obscured AGN population for tighter upper limits. Finally, we measure the X-ray surface brightnesses for our candidate highly-obscured AGN population and others, which we compare with the shape and intensity of the XRB.

The structure of the paper is as follows. In $\S 2$, we describe the radio sample and the available multiwavelength imaging and spectroscopic data. In $\S 3$, we discuss the spectroscopic identifications of the radio sample. In $\S 4$, we briefly describe our photometric redshift method, which uses the optical to MIR SEDs, and we show our redshift distribution for the radio sources. In $\S 5$, we discuss the $24 \mu \mathrm{m}$ properties of the radio sample and the difficulties with using these data to estimate FIR luminosities. In $\S 6$, we describe our spectral classification scheme and examine the properties of the radio sample by spectral class. In $\S 7$, we discuss the X-ray properties of the radio sample and investigate whether there is an X-ray-radio correlation for star-forming galaxies at these higher redshifts, 
like there is locally. In $\S 8$, we describe the optical properties of the radio sample. In $\S 9$, we determine the FIR luminosities of the radio sources using our redshifts and the FIR-radio correlation. We then put quantitative upper limits on the numbers of highly-obscured AGNs that could have gone undetected in this deepest of hard X-ray surveys, and we discuss the implications for the history of supermassive black hole growth. In $\S 10$, we measure the X-ray surface brightnesses of our radio-identified candidate highly-obscured AGN population, as well as of other radio and X-ray populations, and we compare them with the shape and intensity of the XRB. We summarize our results in $\S 11$.

We assume $\Omega_{M}=0.3, \Omega_{\Lambda}=0.7$, and $H_{0}=$ $70 \mathrm{~km} \mathrm{~s}^{-1} \mathrm{Mpc}^{-1}$. All magnitudes are in the AB magnitude system.

\section{THE DATA}

\subsection{Radio and X-ray Data}

Richards (2000) presented a catalog of $1.4 \mathrm{GHz}$ sources detected in the Very Large Array (VLA) ${ }^{5}$ map of the HDF$\mathrm{N}$, which covers a $40^{\prime}$ diameter region with an effective resolution of $1.8^{\prime \prime}$. The absolute radio positions are known to $0.1^{\prime \prime}-0.2^{\prime \prime}$ rms.

Alexander et al. (2003) presented the 2 Ms X-ray image of the CDF-N, which they aligned with the Richards (2000) radio image. Near the aim point, the X-ray data reach limiting fluxes of $f_{2-8} \mathrm{keV} \approx$ $1.4 \times 10^{-16} \mathrm{ergs} \mathrm{cm}^{-2} \mathrm{~s}^{-1}$ and $f_{0.5-2 \mathrm{keV}} \approx 1.5 \times$ $10^{-17} \mathrm{ergs} \mathrm{cm}^{-2} \mathrm{~s}^{-1}$. We determined the maximal radius radio circle that fits within the Chandra area to be a $10^{\prime}$ radius circle centered on the position R.A. $=12^{h} 36^{m} 54.29^{s}$, decl. $=62^{\circ} 14^{\prime} 16.0^{\prime \prime}(\mathrm{J} 2000.0)$. This also roughly matches the highest sensitivity region of the VLA observations, producing a relatively uniform radio map. Our radio sample consists of the 207 radio sources contained within this radius.

For sources within our $10^{\prime}$ radius, matching X-ray counterparts from the Alexander et al. (2003) catalogs to the radio sources is not critically dependent on the choice of match radius. This can be seen from Figure 7 of Alexander et al. (2003), which shows the positional offset between the $\mathrm{X}$-ray and radio sources versus off-axis angle. We chose to use a $1.5^{\prime \prime}$ search radius as a reasonable compromise between not pushing too hard on the accuracy of the data and not introducing too much random error, but only two additional sources would have been matched with a $2^{\prime \prime}$ search radius, and only three of the current sources would not have been matched with a $1^{\prime \prime}$ search radius.

Richards (2000) gave a $5 \sigma$ completeness limit of $40 \mu \mathrm{Jy}$ for compact sources in the central region of the map. The completeness limit is somewhat higher for extended radio sources. We independently determined the completeness of the Richards (2000) $1.4 \mathrm{GHz}$ catalog by measuring the radio fluxes of a large, optically-selected sample in $3^{\prime \prime}$ diameter apertures and adjusting the normalization to match the measured fluxes in the Richards (2000) cata$\log$ for the overlapping sources. Figure 1a shows that the Richards (2000) catalog is highly complete to $70 \mu \mathrm{Jy}$ and then drops to about $50 \%$ completeness at $60 \mu \mathrm{Jy}$.
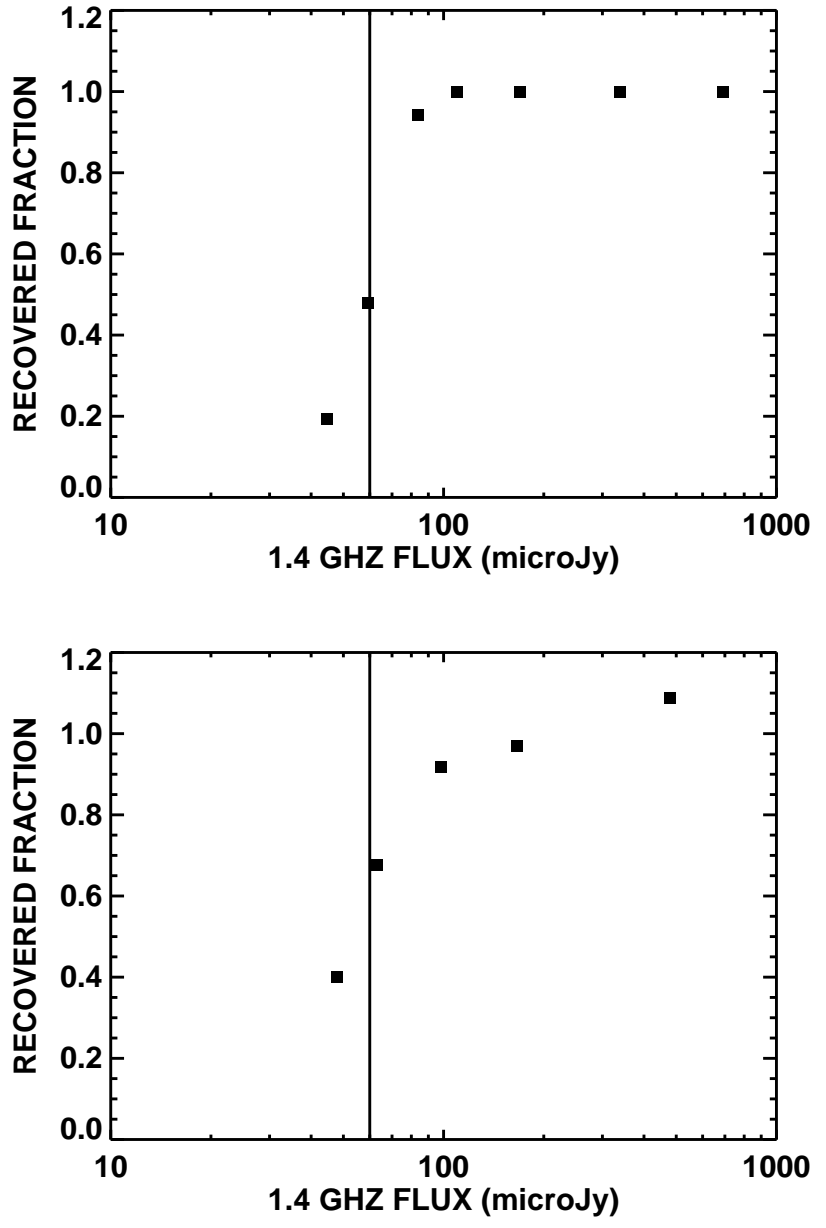

FIG. 1. - Completeness of the HDF-N radio catalog from Richards (2000) as a function of $1.4 \mathrm{GHz}$ flux based on (a) measuring the radio fluxes of an optically-selected sample, and (b) comparing the Richards (2000) catalog with the Biggs \& Ivison (2006) catalog. The solid lines show our adopted $60 \mu \mathrm{Jy}$ completeness limit.

Biggs \& Ivison (2006) have recently given a new cata$\log$ of $1.4 \mathrm{GHz}$ sources using all of the A-array data from the VLA. This catalog is slightly more sensitive than the Richards (2000) catalog and may also be used to estimate the completeness of the Richards (2000) catalog. Within our $10^{\prime}$ radius region, the new catalog contains 280 sources, of which 259 have fluxes greater than $40 \mu \mathrm{Jy}$, as opposed to the 207 sources in the Richards (2000) catalog. Of the 207 Richards (2000) sources, 188 have direct counterparts in the Biggs \& Ivison (2006) catalog. For two other sources, the positions in the two catalogs are offset due to different centerings on the radio sources. The fluxes of the overlapping sources are in reasonable agreement. Most of the 17 Richards (2000) sources that are missing in the Biggs \& Ivison (2006) catalog have optical counterparts and redshifts, so they are probably real and have just dropped below the flux limit of the Biggs \& Ivison (2006) catalog. In Figure 1b, we show the completeness of the Richards (2000) catalog based on the Biggs \& Ivison (2006) catalog. The plot shows the ratio of the number of sources per flux bin detected in the Richards (2000) catalog to the number of sources per flux bin detected in the Biggs \&

\footnotetext{
${ }^{5}$ The VLA is a facility of the National Radio Astronomy Observatory (NRAO). The NRAO is a facility of the National Science Foundation operated under cooperative agreement by Associated Universities, Inc.
} 
Ivison (2006) catalog. Consistent with our analysis of the completeness of the Richards (2000) catalog using the optical data, this shows that the Richards (2000) sample is substantially complete above $70 \mu \mathrm{Jy}$ but becomes progressively more incomplete at lower fluxes. In our subsequent analysis, we adopt $60 \mu \mathrm{Jy}$ as our effective completeness limit.

\subsection{Optical and Near-Infrared Data}

Giavalisco et al. (2004) presented HST ACS images of the HDF-N region (the GOODS-N), and Capak et al. (2004) presented ground-based deep optical imaging of a very wide-field region encompassing the GOODS-N. The ground-based data cover the whole MIPS and IRAC areas (§2.3) in the $U, B, V, R, I, z^{\prime}$, and $H K^{\prime}$ bands. All of the images were registered to the Richards (2000) radio catalog for accurate absolute astrometry.

Trouille et al. (2006, in preparation) carried out deep $J$ - and $H$-band imaging of the entire GOODS-N region using the Ultra-Low Background Camera (ULBCAM) on the University of Hawaii $2.2 \mathrm{~m}$ telescope during 2004 and 2005. ULBCAM consists of four $2 \mathrm{k} \times 2 \mathrm{k}$ HAWAII- 2 RG arrays (Loose et al. 2003) with a total $16^{\prime} \times 16^{\prime}$ field of view. The images were taken using a 13-point dither pattern with $\pm 30^{\prime \prime}$ and $\pm 60^{\prime \prime}$ dither steps in order to cover the chip gaps. The near-infrared (NIR) data were flattened using median sky flats from each dither pattern. The image distortion was corrected using the astrometry in the USNO-B1.0 catalog (Monet et al. 2003). The flattened, sky-subtracted, and warped images (with typical seeing $0.7^{\prime \prime}$ ) were combined to form the final mosaic, which has a $20^{\prime} \times 20^{\prime}$ area that fully covers the GOODS-N region. The final image was registered to the Richards (2000) radio catalog. The integration times at each pixel are 9 hours in $J$ and 12.5 hours in $H$, respectively, and the $5 \sigma$ sensitivities are $0.84 \mu \mathrm{Jy}$ and $2.06 \mu \mathrm{Jy}$, corresponding to $5 \sigma \mathrm{AB}$ magnitude limits of 24.1 and 23.1, respectively.

We measured all of the optical and NIR magnitudes at the positions of the radio sources using $3^{\prime \prime}$ diameter apertures and corrected them to approximate total magnitudes using an average offset for each waveband (Cowie et al. 1994).

\subsection{Mid-Infrared Data}

Wang et al. (2006) combined the reduced DR1 and DR2 IRAC superdeep images from the GOODS-N Spitzer Legacy Science Program first, interim, and second data release products (DR1, DR1+, DR2; Dickinson et al. 2006, in preparation), weighted by exposure time, to form 3.6, $4.5,5.8$, and $8.0 \mu \mathrm{m}$ images that fully cover the GOODS$\mathrm{N}$ area. We measured the source fluxes at the radio positions using fixed 4".8 (3.6 and $4.5 \mu \mathrm{m}$ ) and $6^{\prime \prime}$ (5.8 and $8.0 \mu \mathrm{m})$ diameter apertures. These apertures are approximately three times the $\sim 1^{\prime \prime} .7(3.6 \mu \mathrm{m})$ to $\sim 2^{\prime \prime}(8.0 \mu \mathrm{m})$ FWHM of the point spread function (PSF) and are a good compromise between the PSF size and the source separation. We applied aperture corrections from the IRAC in-flight PSFs (January 2004), which are consistent with the ones published in the IRAC Data Handbook, to the measured fluxes. The corrected IRAC fluxes should be reasonably close to the total fluxes of the sources, because the majority of the sources are point-like compared to the $\sim 2^{\prime \prime}$ IRAC PSF. The primary errors in the photometry are caused by the high density of sources, especially at 3.6 and $4.5 \mu \mathrm{m}$. In these two bands, the typical distance between sources is comparable to the PSF, and the maps are confusion-limited. Consequently, both the background estimate and the aperture photometry are highly subject to blending with nearby sources.

Also following Wang et al. (2006), we directly used the DR1+ MIPS $24 \mu \mathrm{m}$ source list and version 0.36 MIPS $24 \mu \mathrm{m}$ map provided by the Spitzer Legacy Program. This source catalog is flux-limited at $80 \mu \mathrm{Jy}$ and is a subset of a more extensive catalog (Chary et al. 2006, in preparation). With the $0.065 \mathrm{deg}^{2}$ area coverage, the catalog contains $119924 \mu \mathrm{m}$ sources and is highly complete at $80 \mu \mathrm{Jy}$. The source positions are based on sources detected in the deep IRAC images, and the fluxes are derived using PSF fitting. A $-00^{\prime \prime} 38$ offset in declination was applied to the source positions to match the radio-frame astrometry (Richards 2000). We cross-identified the MIPS sources with the radio sources by searching a $1.5^{\prime \prime}$ radius region around the radio positions.

\subsection{Spectroscopic Data}

Cowie et al. (2004b) and Wirth et al. (2004) report the results of an extensive spectroscopic survey of galaxies in the ACS GOODS-N region. We supplemented these data with spectral observations made with the Deep Extragalactic Imaging Multi-Object Spectrograph (DEIMOS; Faber et al. 2003) on the Keck II $10 \mathrm{~m}$ telescope subsequent to the publication of these papers and with targeted observations of radio and X-ray sources (Barger et al. 2002, 2003, 2005; Cowie et al. 2004a) made with either DEIMOS or the Low-Resolution Imaging Spectrograph (LRIS; Oke et al. 1995) on the Keck I telescope. We also use redshifts obtained by Chapman et al. $(2004,2005)$ and Swinbank et al. (2004), adopting the Swinbank et al. (2004) NIR redshifts over the Chapman et al. (2004, 2005) optical redshifts, where available, because redshift measurements are generally more reliable when they are made from emissionline features. Where there are redshifts from both sources, the redshifts are within $<0.008$ of each other.

\section{RADIO SAMPLE IDENTIFICATIONS}

Cross-identification of the radio sources with their counterparts is non-trivial. Some radio sources have complex structures, and it is only by inspection of overlays of the radio contours on optical through MIR images that the likely counterparts can be identified. In other cases, the counterparts are extremely red, only emerging in the NIR or MIR bands. (Reassuringly, these red cores inevitably agree with the radio positions.) The most extreme of these sources can only be identified in the Spitzer IRAC bands, which do not cover the entire radio field.

There are also cases where previous spectroscopic identifications (found through a search of the literature via the NASA/IPAC Extragalactic Database, NED) appear to be of neighboring, usually much bluer galaxies, rather than of the genuine counterparts. It is possible that some of these identifications may, in fact, be correct if the bluer structures are parts of a more extended galaxy centered on the red core. However, it is very hard to make a convincing argument that this is indeed the case, rather than, for example, that the blue source is a background galaxy lensed by the foreground red source. 
Here we try to take a conservative approach to the identifications. We first determined which sources have unambiguous counterparts. These include all of the sources which have either a $5 \sigma$ ground-based $z^{\prime}$ - or $H$-band counterpart or an IRAC $5.4 \mu \mathrm{m}$ counterpart lying within $1^{\prime \prime}$ of the radio position. This immediately identifies 195 of the sources in the sample. The dispersion of these sources around the radio positions is $0.3^{\prime \prime}$.

We then overlaid the radio contours of the remaining 12 sources on the optical images of the field (Fig. 2). One source, with a flux of $5.96 \mathrm{mJy}$, is clearly bi-lobal and centered on a small red galaxy. We take this red galaxy to be the counterpart to the radio source. The remaining 11 sources have no obvious identifications, though three lie near bright galaxies (one of these could be an off-axis source; see below), and a fourth may be associated with a pair of interacting galaxies at $z=0.642$. Nearly all of these are faint sources $(40-80 \mu \mathrm{Jy})$, and some may be false. (Note that Richards 2000 found a $-9 \sigma$ source within the HDF-N radio image, and since the probability of finding such a source in the image is much less than $1 \%$, he concluded that the noise properties of the image are not entirely Gaussian.) However, four of the remaining 11 sources have fluxes above $70 \mu \mathrm{Jy}$, and four (including only one of the sources with a flux above $70 \mu \mathrm{Jy}$ ) are in the deeper Biggs \& Ivison (2006) catalog (see Table 1). These may be real sources without counterparts from the optical to the MIR. For example, they could be very obscured or perhaps high-redshift galaxies.

FIG. 2.- Mosaic of the bi-lobal source and the 11 remaining sources in our radio sample without obvious counterparts. The sources are shown in the order of Table 1, starting from the bottomleft corner and moving to the right, then looping back to the beginning of the next row and again moving to the right. The individual images are $12.5^{\prime \prime}$ on a side. There are three colors in the images: blue is set to be the $B$ image, green is set to be the $V$ image, and red is set to be the average of $I+z^{\prime}$, all from the ACS GOODS-N data. The contours show the positions of the radio light.

FIG. 3. - Mosaic of the 12 sources whose redshifts we omitted due to the uncertainty of whether these literature identifications are directly associated with the radio sources. The sources are shown in the order of Table 2, starting from the bottom-left corner and moving to the right, then looping back to the beginning of the next row and again moving to the right. The individual images are $12.5^{\prime \prime}$ on a side. There are three colors in the images: blue is set to be the $B$ image, green is set to be the $V$ image, and red is set to be the average of $I+z^{\prime}$, all from the ACS GOODS-N data. The contours show the positions of the radio light.

We summarize the radio and optical coordinates of the bi-lobal source and the radio coordinates of the 11 sources without obvious conterparts in Table 1. Only one of these sources has an X-ray or $24 \mu \mathrm{m}$ detection. This source lies in the outer regions of a bright $z=0.459$ galaxy and may be either an off-axis source in that galaxy or a faint background source that is hidden by the foreground galaxy and hence cannot be identified.

Next, we determined which of the sources with unambiguous counterparts had spectroscopic redshifts. We omitted redshifts for 12 sources with identifications in the literature (from NED), where inspection of the images suggested that the identifications may not be directly associated with the radio sources. As we have noted above, some of these identifications may be correct, but it appears safer to rely on the photometric redshifts for these sources. We summarize the omitted sources in Table 2, and we show the radio contours overlaid on the optical images for these sources in Figure 3.

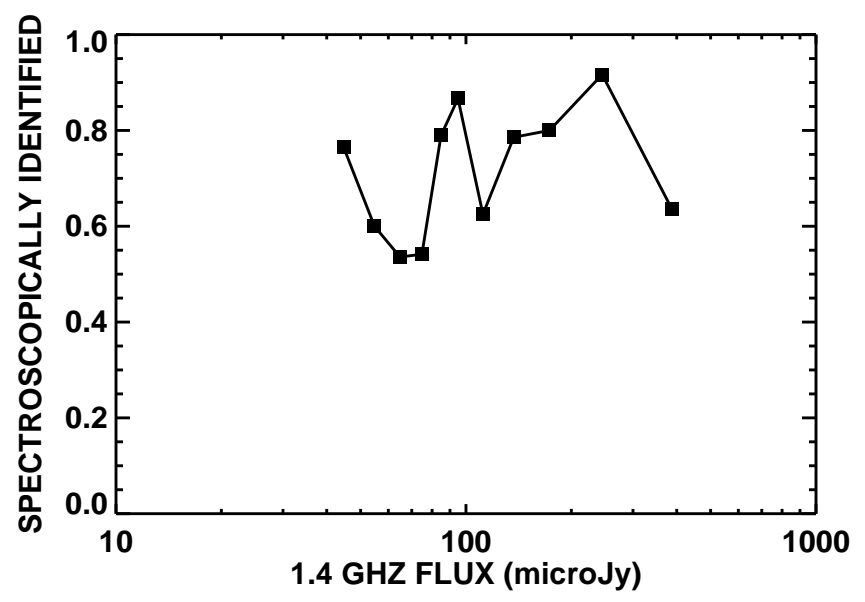

FIG. 4.- Spectroscopic completeness of our radio sample. The sources are grouped into the following flux bins: 40-50, 50-60, 6070, 70-80, 80-90, 90-100, 100-125, 125-150, 150-200, 200-300, 300500. All of these bins contain 10 or more sources.

Our final compilation contains 143 spectroscopic redshifts, of which 101 are already in the literature, and 42 are new to this work. In our subsequent analysis, we distinguish the 12 redshifts that we use from Chapman et al. (2004, 2005) and Swinbank et al. (2004) from our other redshifts, referring to them as the CS sources. In Figure 4 , we show the fraction of radio sources that are spectroscopically identified. We see that the identified fraction is fairly independent of radio flux, with about $60-80 \%$ of the sources identified at all fluxes.

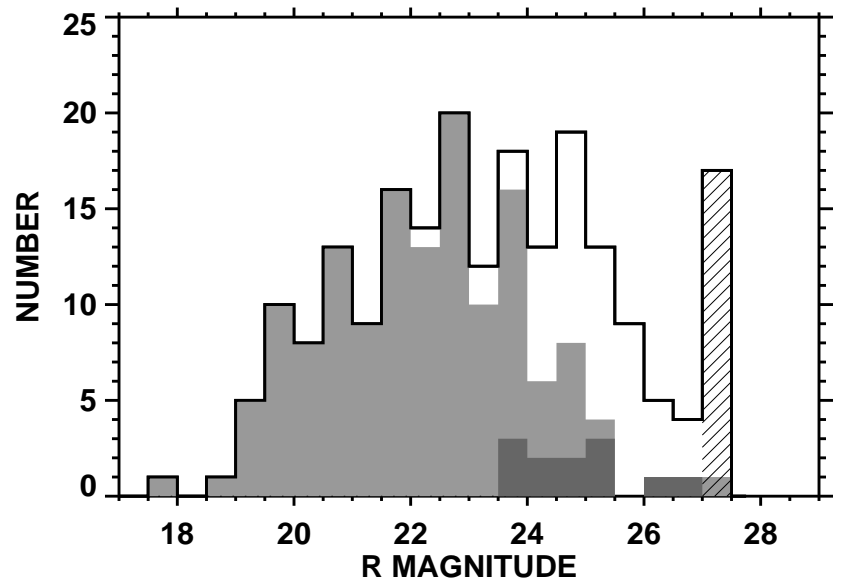

FIG. 5.- $R$ magnitude distribution for all of the sources in our radio sample (open). Shading denotes the spectroscopically identified sample, with darker shading for the CS sources. All seventeen $R>27$ sources are placed in the final magnitude bin (hatched). Sources with $R<20$ suffer from saturation problems and are likely to be brighter than measured.

In Figure 5, we show the $R$ magnitude distribution for all of the sources in our radio sample (open). We denote the spectroscopic sample with light shading and the CS sources with dark shading. Beyond $R=24$, it becomes more difficult to spectroscopically identify the radio sources, while at brighter magnitudes, essentially all of the sources can be identified. 


\section{PHOTOMETRIC REDSHIFTS}

Wang et al. (2006), using the method of Pérez-González et al. (2005), built "training-set" SEDs from 1200 galaxies in the MIPS GOODS-N sample with known redshifts and spectral types. They built seven templates, ranging from an elliptical galaxy spectrum to a very blue starforming galaxy spectrum, over the frequency range from $6 \times 10^{13} \mathrm{~Hz}$ to $4 \times 10^{15} \mathrm{~Hz}$. They then made least-squares fits of their individual source SEDs to these templates to determine the photometric redshifts and spectral types for the galaxies in their samples. They found that the method worked extremely well over a wide range of redshifts and only failed for a small number of sources. Their use of the deep $J$ - and $H$-band magnitudes and the Spitzer data, in addition to the optical data, substantially improved the high-redshift end of the photometric redshift determinations.

To derive photometric redshifts for our radio sources, we similarly constructed SEDs using the optical data, the Spitzer IRAC $3.6 \mu \mathrm{m}, 4.5 \mu \mathrm{m}, 5.8 \mu \mathrm{m}$, and $8.0 \mu \mathrm{m}$ data, the Spitzer MIPS $24 \mu \mathrm{m}$ data, and the ULBCAM NIR $J$ and $H$-band data. We then made least-squares fits to the Wang et al. (2006) templates to determine the photometric redshifts and spectral types for the galaxies in our radio sample that were detected in at least five bands. This gives us an additional 47 redshifts. Thus, in total, only 17 of our radio sources (including the 11 without any obvious counterparts) have neither a spectroscopic nor a photometric redshift. We show a comparison of the photometrically identified sources (open) with the spectroscopically identified sources (light shading) in Figure 6. The CS sources are denoted by dark shading. The gap in the spectroscopic redshift distribution between $z \sim 1.4$ and $z \sim 1.9$, known as the "redshift desert", reflects the difficulty of identifying galaxies with redshifts in this range, where [OII] $3727 \AA$ has moved out of the optical window and Ly $\alpha 1216 \AA$ has not yet entered in.

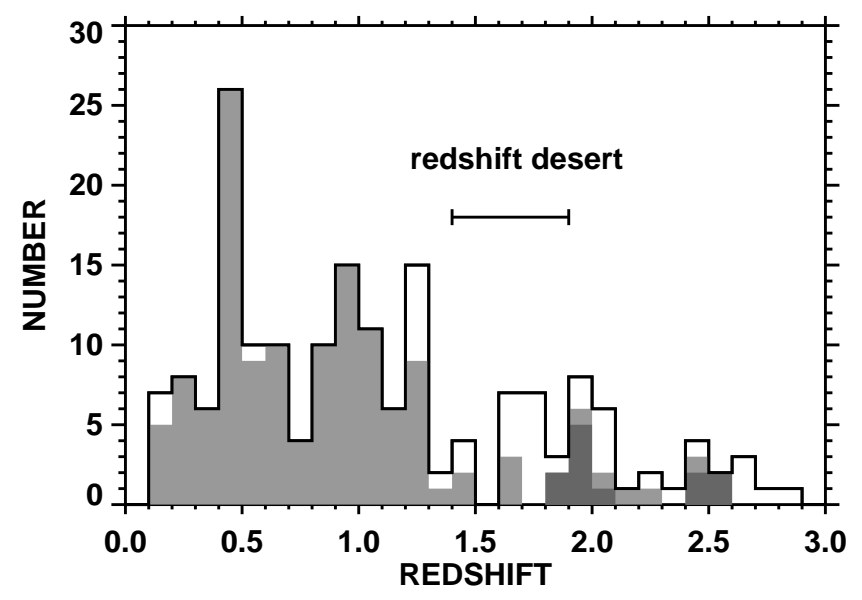

FIG. 6.- Number of spectroscopically (shaded) and photometrically (open) identified sources vs. redshift for the radio sample. The CS sources are denoted by dark shading.

\section{24 MICRON PROPERTIES OF THE RADIO SAMPLE}

Studies based on the Infrared Space Observatory (ISO) $15 \mu \mathrm{m}$ data found a loose correlation between the MIR emission and the radio continuum (Cohen et al. 2000; El- baz et al. 2002; Garrett 2002; Gruppioni et al. 2003). Appleton et al. (2004) confirmed the existence of this correlation with Spitzer data, and Marcillac et al. (2006) proposed using it to derive FIR luminosities. However, while obtaining the FIR luminosity from a radio measurement only depends on the FIR-radio correlation and the radio spectral index (and the assumption that the source is not a radio-loud $\mathrm{AGN}$ ), obtaining the FIR luminosity from a MIR measurement strongly depends on the library of template SEDs that is used to $K$-correct the data.

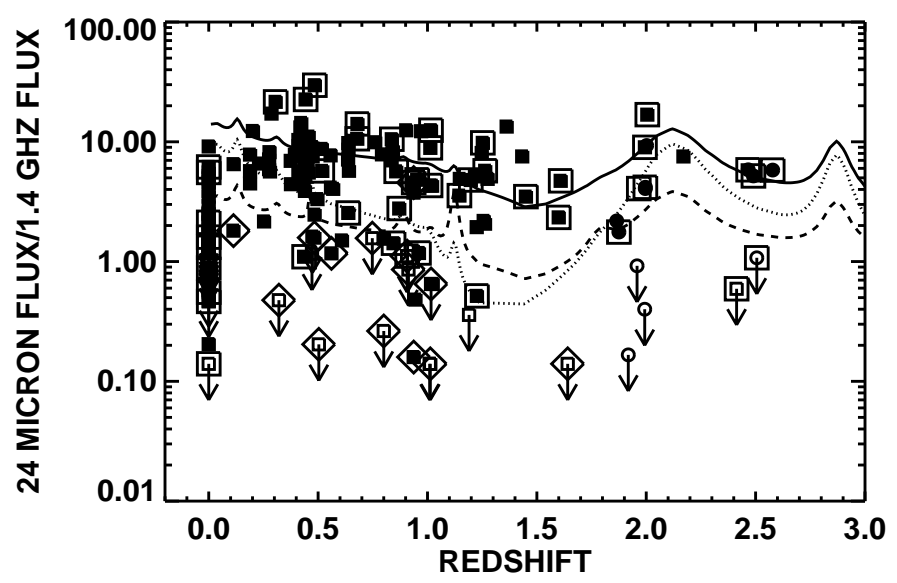

FIG. 7. - Ratio of $24 \mu \mathrm{m}$ flux to $1.4 \mathrm{GHz}$ flux vs. redshift for the spectroscopically identified radio sources in the MIPS area. Solid (open) symbols denote sources with (without) $24 \mu \mathrm{m}$ detections. Circles denote the CS sources. Large open squares denote sources

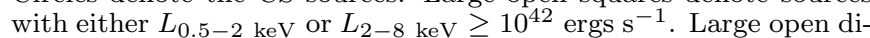
amonds denote spectroscopically classified absorbers. Spectroscopically unidentified sources are nominally plotted at $z=0$. The overplotted curves are the ratios from redshifted template SEDs of M82 (solid), Arp 220 (dotted), and a spiral galaxy (dashed) (Silva et al. 1998).

We illustrate the difficulty with using MIR data to estimate FIR luminosities in Figure 7 (similar to Figure 1 of Donley et al. 2005), where we show the observed $24 \mu \mathrm{m}$ to $1.4 \mathrm{GHz}$ flux ratio versus redshift. Solid (open) symbols denote sources with (without) $24 \mu \mathrm{m}$ detections, and large open squares denote sources with soft or hard X-ray luminosities in excess of $10^{42} \mathrm{ergs} \mathrm{s}^{-1}$. For the sources without $24 \mu \mathrm{m}$ detections, we have adopted the $80 \mu \mathrm{Jy}$ flux limit of the $24 \mu \mathrm{m}$ source catalog (see $\S 2.3$ ). We have overplotted $24 \mu \mathrm{m}$ to $1.4 \mathrm{GHz}$ flux ratios versus redshift for redshifted template SEDs (Silva et al. 1998). It is clear that there is substantial spread in the ratio, with the $24 \mu \mathrm{m}$ measurements being very sensitive both to redshift and to galaxy type. For the most part, the data points have values that are within the range expected from the redshifted template SEDs; however, there are also a number of sources not detected at $24 \mu \mathrm{m}$, including many of the absorbers (large open diamonds; see $\S 6$ ). The absorbers are most likely radio-loud AGNs, which would not be expected to have SEDs like those shown.

We note that Donley et al. (2005), in their search for highly-obscured AGNs, defined a selection threshold of $q=\log \left(S_{24 \mu \mathrm{m}} / S_{1.4 \mathrm{GHz}}\right)<0$ to classify a galaxy as probably having an AGN. Based on the SEDs used in our Figure 7 , this criterion might result in some contamination by ultraluminous infrared galaxies, and, to a lesser extent, by spirals, as both of those SEDs dip below $q=0$. We do 
see sources below this value that do not have X-ray luminosities typical of an AGN and are not spectroscopically classified as absorbers, and some of these sources may be highly-obscured AGNs, but clearly we cannot rely on the $24 \mu \mathrm{m}$ data to determine their FIR luminosities.

\section{PROPERTIES OF THE RADIO SAMPLE BY SPECTRAL CLASS}

For our spectroscopically identified sources, we classified the optical spectra into four spectral classes, roughly following the procedure used by Sadler et al. (2002) to analyze low-redshift $1.4 \mathrm{GHz}$ samples. We classified sources without any strong emission lines $[\mathrm{EW}([\mathrm{OII}])<3 \AA$ or $\mathrm{EW}(\mathrm{H} \alpha+\mathrm{NII})<10 \AA)]$ as absorbers; sources with strong Balmer lines and no broad or high-ionization lines as star formers; sources with [NeV] or CIV lines or strong [OIII] $[\mathrm{EW}([\mathrm{OIII}] 5007 \AA)>3 \mathrm{EW}(\mathrm{H} \beta)]$ as Seyfert galaxies, and, finally, sources with optical lines having FWHM line widths $>2000 \mathrm{~km} \mathrm{~s}^{-1}$ as broad-line AGNs. We have not classified one high-redshift source at $z=2.2032$. This source must be a strong star former because it has UV absorption lines; however, its [OII] emission is redshifted into the NIR and hence cannot be measured with our spectra. We have also not attempted to spectroscopically classify the CS sources, which are generally of this type.

\subsection{Radio-to-Optical Ratios by Spectral Class}

Some radio quasar studies have found bimodal distributions of rest-frame radio-to-optical ratios (e.g., Kellermann et al. 1989; Stocke et al. 1992; Ivezić et al. 2002; however, see White et al. 2002; Cirasuolo et al. 2003). These have been interpreted as evidence that quasars come in two distinct populations, 'radio-loud' and 'radio-quiet'. The boundary value between radio-loud and radio-quiet has been inferred to be $f_{5 \mathrm{GHz}} / f_{2500 \AA} \stackrel{\circ}{=} 10$ (Stocke et al. 1992).

For our study, we define a rest-frame optical-to-radio ratio of $R=f_{1.4 \mathrm{GHz}} / f_{6500} \stackrel{\circ}{A}$. This is an approximately equivalent ratio, since the $K$-correction to go from $5 \mathrm{GHz}$ to $1.4 \mathrm{GHz}$ is very similar to the $K$-correction to go from $2500 \AA$ to $6500 \AA$, assuming spectral indices of $\alpha=0.8$ for $f_{\nu} \propto \nu^{-\alpha}$ in both cases (Yun et al. 2001; Zheng et al. 1997).

In Figure 8, we show a histogram of logarithmic $R$ for our $z<1.6$ spectroscopically classified radio sample. We have such uniform and complete wavelength coverage for all of the radio sources that we have just interpolated between our measurements to obtain rest-frame AB $6500 \AA$ magnitudes. We have restricted the plot to sources with $z<1.6$ so that our interpolation remains valid. We have also $K$-corrected the radio measurements to rest-frame $1.4 \mathrm{GHz}$. The star formers clearly dominate the microJansky sample, as was inferred from previous work (e.g., Windhorst et al. 1995; Richards et al. 1998; Richards 2000; Roche et al. 2002).

Unfortunately, we do not have a large enough sample of Seyfert galaxies plus broad-line AGNs to investigate whether there is bimodality in the rest-frame radio-tooptical ratios. However, we can see that all of the spectral classes show a wide range in radio-to-optical ratios. Thus, in contrast to the conclusions of Machalski \& Condon (1999) from their study of milliJansky radio sources in the Las Campanas Redshift Survey, we do not find this ratio to be a useful discriminator between star-forming galaxies and AGNs. Afonso et al. (2005) came to a similar conclusion using the Phoenix Deep Survey, which reaches into the sub-100 $\mu \mathrm{Jy}$ regime. They argued that the radio morphology and polarimetry used by Machalski \& Condon (1999) to identify AGNs results in the misclassification of many low-power AGNs, and these sources would blur the distinction if properly classified as AGNs. Since the radioto-optical ratio does not appear to have much diagnostic power for our sample, we do not use it subsequently.

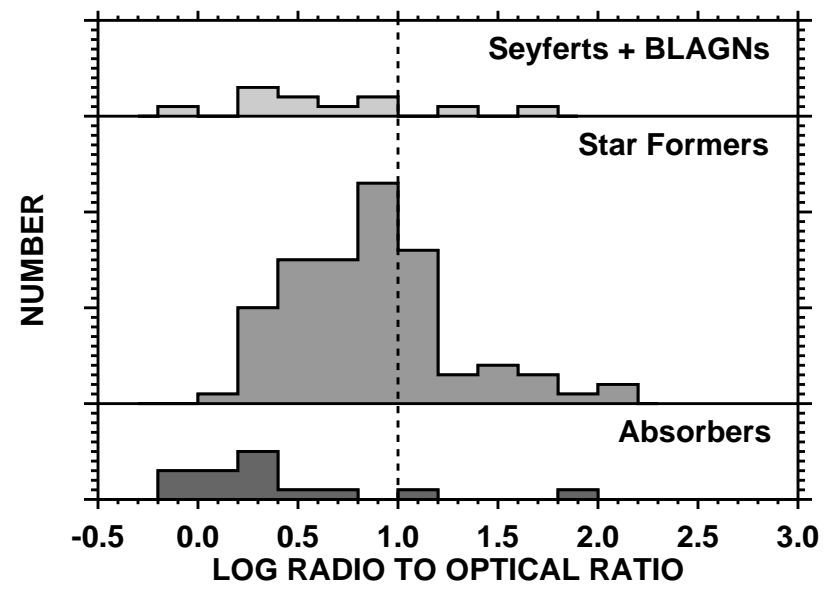

FIG. 8. - Histogram of the logarithmic rest-frame radio-tooptical ratio, $R$, for our spectroscopically classified $z<1.6$ radio sample divided into absorbers, star formers, and Seyfert galaxies plus broad-line AGNs (BLAGNs). Each tickmark on the y-axis represents one source. The dashed vertical line shows the classical boundary $(R=10)$ between radio-quiet and radio-loud.

\subsection{Radio Power and Redshifts by Spectral Class}

In Figure 9, we show histograms of the (a) logarithmic radio powers and (b) redshifts for the spectroscopically classified radio sample (shaded). We calculate the restframe radio powers from the equation

$$
P_{1.4 \mathrm{GHz}}=4 \pi d_{L}{ }^{2} S_{1.4 \mathrm{GHz}} 10^{-29}(1+z)^{\alpha-1} \mathrm{ergs} \mathrm{s}^{-1} \mathrm{~Hz}^{-1} \text {. }
$$

Here $d_{L}$ is the luminosity distance $(\mathrm{cm}), S_{1.4 \mathrm{GHz}}$ is the $1.4 \mathrm{GHz}$ flux density $(\mu \mathrm{Jy})$, and $\alpha$ is the radio spectral index, which we take to be 0.8 (Yun et al. 2001).

The bulk of the star formers tend to lie at the lower radio powers. The redshift distribution for the Seyfert galaxies plus broad-line AGNs stretches to higher redshifts than the star formers and the absorbers, though the absorbers in particular become more difficult to identify spectroscopically at $z>1$. We show that this is a selection effect by including on Figure $9 \mathrm{~b}$ the redshift distribution for the photometrically identified absorbers classified using their SEDs (see §4) (open).

\section{X-RAY PROPERTIES OF THE RADIO SAMPLE}

An X-ray-radio correlation for star-forming galaxies has been determined locally by a number of authors (e.g., Bauer et al. 2002; Ranalli et al. 2003; Grimm, Gilfanov, \& Sunyaev 2003; Gilfanov, Grimm, \& Sunyaev 2004), suggesting that the X-ray emission in star-forming galaxies can be used as an indicator of the star formation rate. 
These same authors, using a small sample of sources in the CDF-N (at the time, only the $1 \mathrm{Ms}$ Chandra data were available; Brandt et al. 2001), also claim that the linear relation extends to higher redshifts $(z<1.3)$. With our highly spectroscopically complete, cleanly selected sample from just one flux band $(1.4 \mathrm{GHz})$, we are in an excellent position to test whether the local X-ray-radio correlation does indeed continue to hold to higher redshifts.
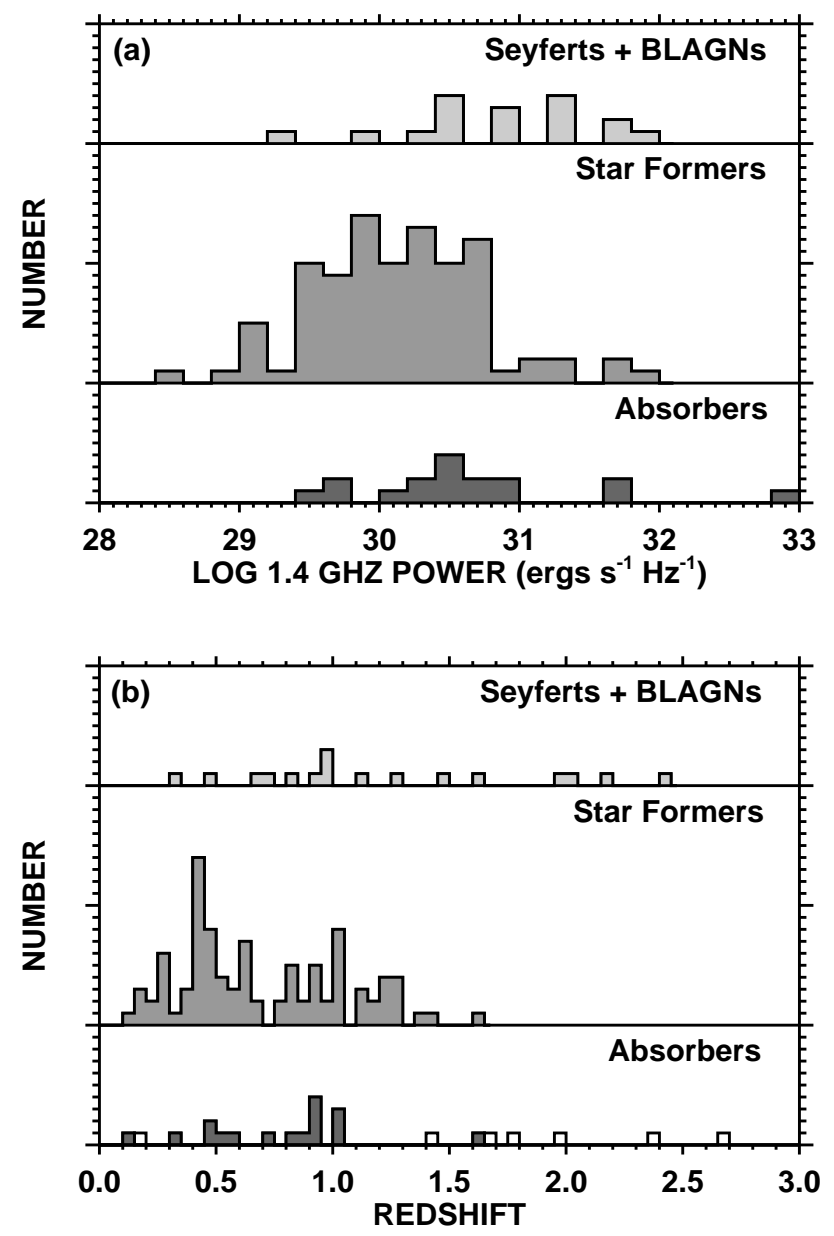

FIG. 9.- (a) Logarithmic power and (b) redshift distributions for our spectroscopically classified radio sample (shaded) divided into absorbers, star formers, and Seyfert galaxies plus broad-line AGNs (BLAGNs). Each tickmark on the y-axis represents one source. In (b), we also show the redshift distribution for photometrically identified absorbers classified using their SEDs. (see §4) (open).

In Figure 10a (10b), using different symbols for the different spectral classes, we plot $2-8 \mathrm{keV}(0.5-2 \mathrm{keV})$ luminosity over $1.4 \mathrm{GHz}$ power (we use the ratio to remove the redshift effect and improve the dynamic range) versus 1.4 GHz power for the spectroscopically identified galaxies in our radio sample that were significantly detected by Alexander et al. (2003) in the $2-8 \mathrm{keV}(0.5-2 \mathrm{keV})$ band. We calculated the rest-frame $1.4 \mathrm{GHz}$ powers using Eq. 1, and we calculated the rest-frame hard (soft) X-ray luminosities from

$$
L_{X}=4 \pi d_{L}^{2} f_{X}(1+z)^{\Gamma-2} \operatorname{ergs~s}^{-1} .
$$

Here $d_{L}$ is the luminosity distance $(\mathrm{cm}), f_{X}$ is the observed-frame hard (soft) X-ray flux (ergs $\mathrm{cm}^{-2} \mathrm{~s}^{-1}$ ), and $\Gamma$ is the photon index, which we take to be 1.8 for all of the sources. Note that using the individual photon indices (rather than the universal power-law index of $\Gamma=1.8$ ) to calculate the $K$-corrections would result in only a small difference in the rest-frame luminosities (Barger et al. 2002).

We assume that any source more X-ray luminous than $10^{42} \mathrm{ergs} \mathrm{s}^{-1}$ is very likely to be an AGN on energetic grounds (Zezas et al. 1998; Moran et al. 1999), so we illustrate the ratio of this fixed hard (soft) X-ray luminosity to the $1.4 \mathrm{GHz}$ power versus the $1.4 \mathrm{GHz}$ power with a diagonal line in the figures. We note that since none of the star-forming galaxies (solid diamonds) show any obvious AGN signatures in their optical spectra, those with $L_{X} \geq 10^{42} \mathrm{ergs} \mathrm{s}^{-1}$ are most likely to be obscured AGNs.

With a dashed horizontal line, we show the local hard (soft) X-ray/radio relation found by Ranalli et al. (2003; their Eqs. 13 and 9, respectively). We have converted their $2-10 \mathrm{keV}$ relation to $2-8 \mathrm{keV}$, assuming a photon index of $\Gamma=1.8$. Although in Figure 10a we have too few significantly hard X-ray detected, star-forming galaxies with $L_{2-8} \mathrm{keV}<10^{42} \mathrm{ergs} \mathrm{s}^{-1}$ (large open diamonds) to identify any kind of correlation, in Figure 10b, we can see the correlation that previous authors had identified. However, several radio-powerful star formers significantly detected in soft X-rays lie well below the apparent correlation and may be the luminous edge of the X-ray undetected population. In fact, it is important to stress that the above figures only include X-ray detected sources, not upper limits. Including the upper limits would be the true test of whether a correlation actually exists or whether one is just observing the upper end of the distribution and hence being fooled into thinking that there is a correlation when, in actuality, it is merely a selection effect.

Unfortunately, from Figures 10c and d, we can see that the apparent correlation is indeed merely a selection effect. Here we plot only the spectroscopically identified star formers that are not significantly detected in the $2-8 \mathrm{keV}$ and $0.5-2 \mathrm{keV}$ bands, respectively, using the hard and soft X-ray detection limits from Alexander et al. (2003) (downward-pointing arrows). The diagonal and horizontal lines are as before. The first thing to note is that even with the $2 \mathrm{Ms}$ exposure, we do not have the sensitivity to measure the bulk of the star-forming galaxy population in $\mathrm{X}$-rays. The second thing to note is that-again seen most clearly in the soft X-ray figure - the upper limits are not consistent with the Ranalli et al. (2003) relation.

We note that some of the sources in Figure 10 may be radio-loud AGNs, but anyone using the supposed correlation with their X-ray data is going to be faced with that uncertainty. Thus, the fact that we do not confirm the existence of an X-ray-radio correlation at these redshifts, even with our knowledge of the optical spectra of the sources, is a major concern for those who want to infer star formation rates for their galaxies. Although there are attractions to measuring a star formation history based on X-ray surveys because it avoids problems of obscuration (e.g., Norman et al. 2004), we are forced to conclude that it is not easy to do so.

\section{OPTICAL PROPERTIES OF THE RADIO SAMPLE}

In Figure 11a, we plot $1.4 \mathrm{GHz}$ flux versus $R$ magnitude for the radio sample. Interestingly, there is no observed correlation between $1.4 \mathrm{GHz}$ flux and $R$ magnitude, which 

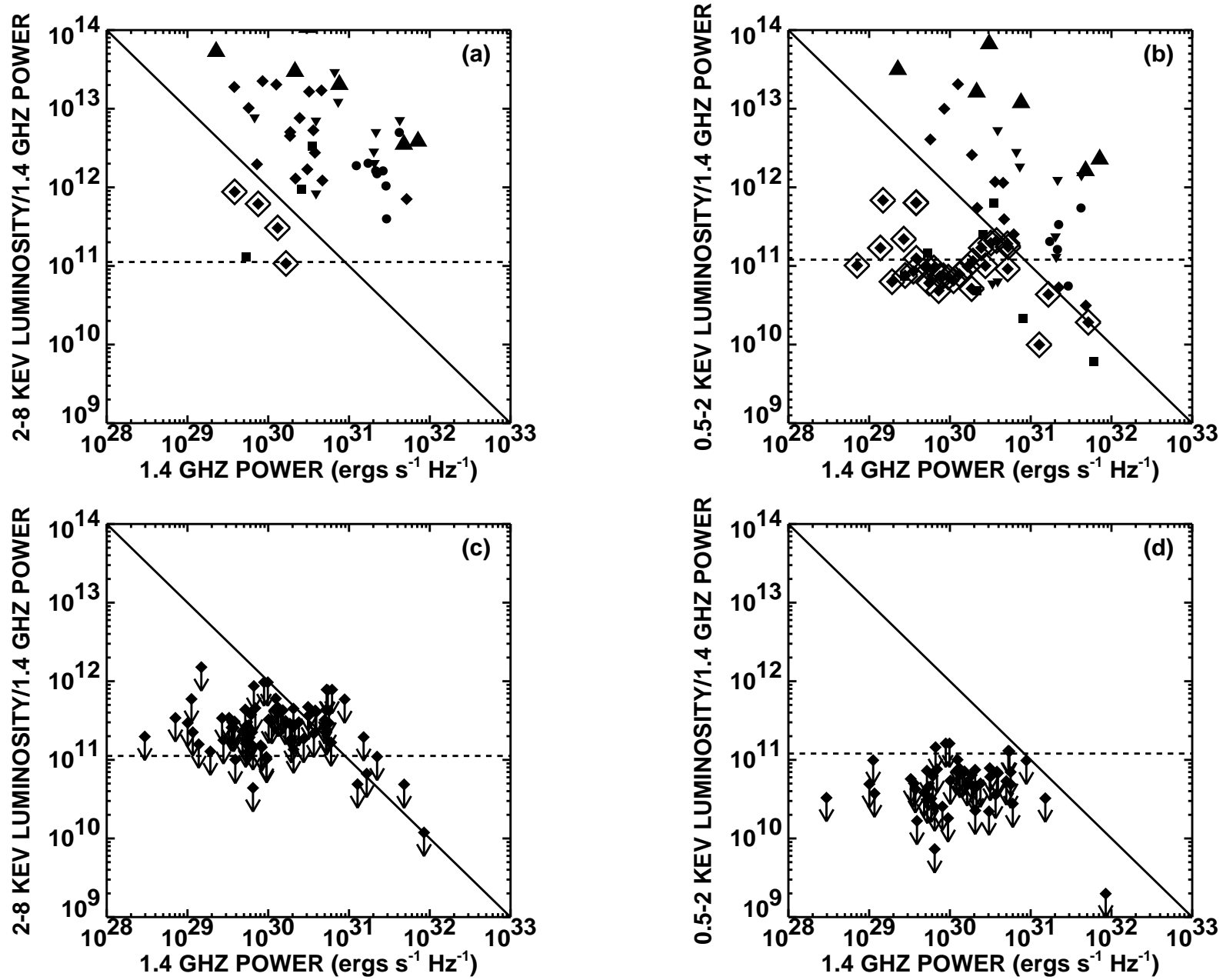

Fig. 10.- Rest-frame $2-8 \mathrm{keV}$ or $0.5-2 \mathrm{keV}$ luminosity over $1.4 \mathrm{GHz}$ power vs. $1.4 \mathrm{GHz}$ power for the spectroscopically identified galaxies in the radio sample (absorbers-solid squares; star formers-solid diamonds; Seyfert galaxies-solid upside-down triangles; broadline AGNs - large, solid triangles; CS sources and our unclassified $z=2.2032$ source [see §2.4] - solid circles) that are (a) significantly hard X-ray detected, (b) significantly soft X-ray detected, (c) star-forming galaxies only without significant hard X-ray detections, (d) star-forming galaxies only without significant soft X-ray detections. In (a) and (b), the large, open diamonds denote significantly X-ray detected, star-

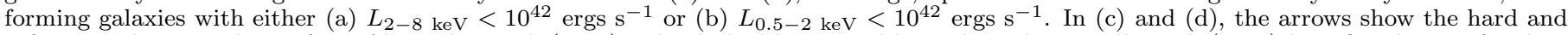
soft X-ray detection limits from Alexander et al. (2003). The dashed horizontal lines show the Ranalli et al. (2003) best-fit relations for their local sample assuming a linear slope (their Eqs. 9 and 13 for the soft and hard X-ray bands, respectively; we have $K$-corrected the latter to $2-8 \mathrm{keV}$ from $2-10 \mathrm{keV}$ assuming $\Gamma=1.8$ ). The solid diagonal lines assume a fixed X-ray luminosity of $10^{42} \mathrm{ergs} \mathrm{s}^{-1}$, the luminosity above which the sources are most certainly powered by AGNs. 
helps to explain the uniformity of the spectroscopically identified fraction with radio flux (see Fig. 4). We illustrate this in histogram form in Figure 11b, where we plot number versus $R$ magnitude for all of the radio sources in our sample, divided into five radio flux bins. The $R$ magnitude distribution is consistent with being drawn from an invariant population. This result differs from that of Georgakakis et al. (1999) and Afonso et al. (2005), who found fainter median $R$ magnitudes with decreasing radio flux density. The most likely reason for the discrepancy is that the optical observations used by those authors were much shallower, probing only to $R=22.5$.
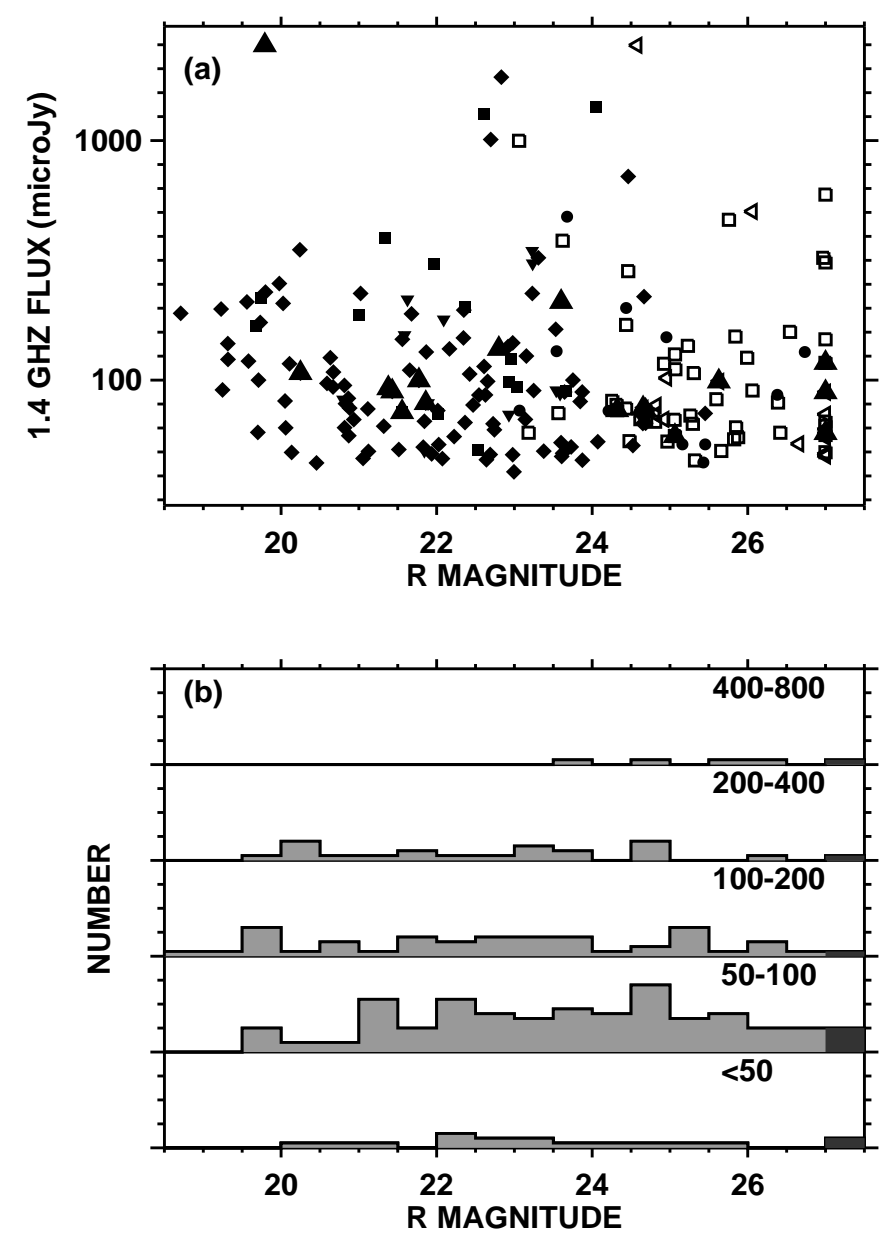

Fig. 11.- (a) $1.4 \mathrm{GHz}$ flux vs. $R$ magnitude for the radio sample (absorbers-solid squares; star formers-solid diamonds; Seyfert galaxies - solid upside-down triangles; broad-line AGNslarge, solid triangles; CS sources and our unclassified $\mathrm{z}=2.2032$ source [see §2.4] - solid circles; photometric redshifts-open squares; unidentified sources-open leftward-pointing triangles). Sources with radio fluxes greater than $2500 \mu \mathrm{Jy}$ are shown at that flux, and sources with $R>27$ are shown at that magnitude. Sources with $R<20$ suffer from saturation problems and are likely to be brighter than measured. Radio flux ranges in microJanskys are given for each bin. Each tickmark on the $y$-axis represents five sources. Darker shading denotes sources with $R>27$.

In Figure 12a, we show radio flux versus redshift for the spectroscopically (solid squares) and photometrically (open squares) identified sources in the radio sample. We plot the unidentified sources at $z<0$. Again we see no correlation between the two variables. In Figure 12b we show this in histogram form, plotting number versus redshift for all of the radio sources with either spectroscopic or photometric redshifts, divided into five radio flux bins. The redshift distribution is consistent with being invariant with radio flux, although the selection of optically bright sources through the spectroscopic identification process may mean that we are missing the higher redshift tail of the redshift distribution function. Thus, probing to fainter radio fluxes does not sample to higher redshifts (Condon 1989).
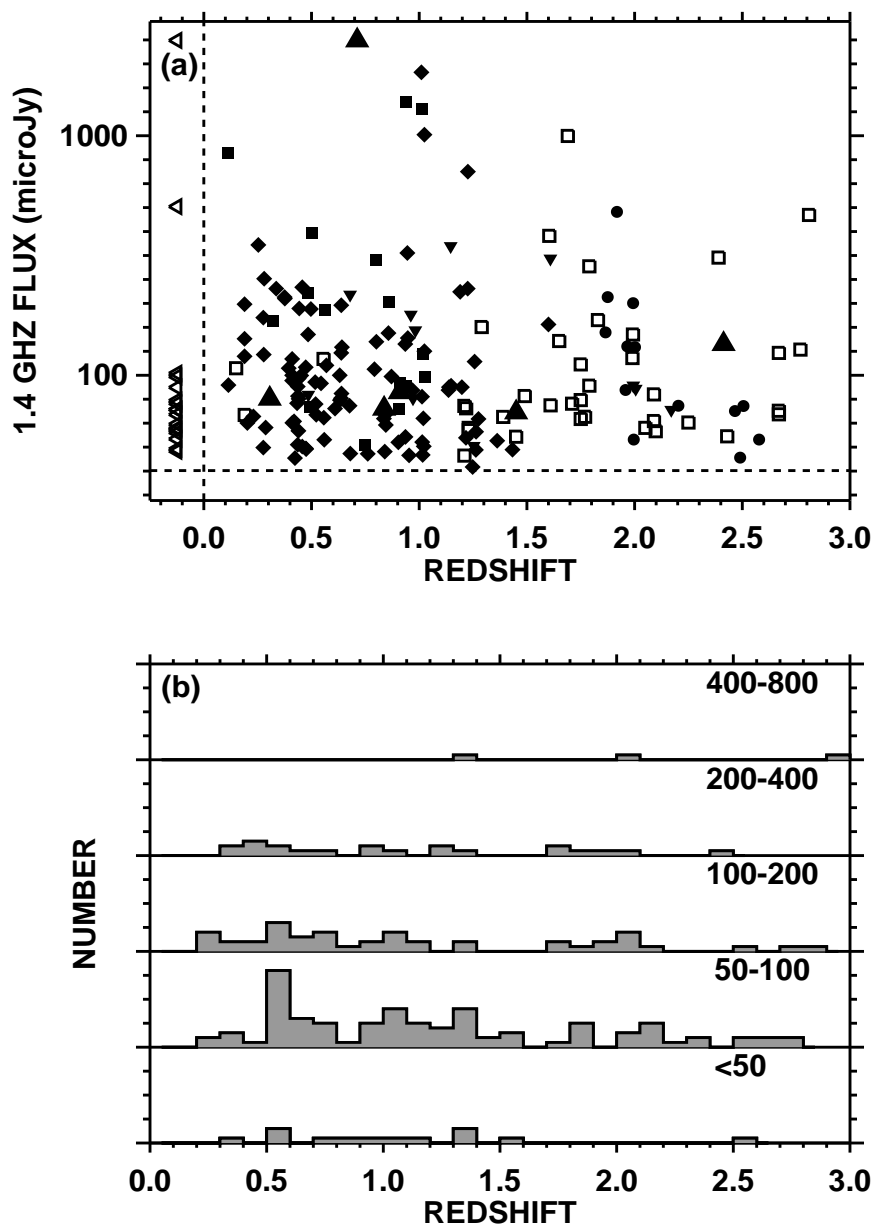

FIG. 12.- (a) $1.4 \mathrm{GHz}$ flux vs. redshift for the radio sample (absorbers-solid squares; star formers-solid diamonds; Seyfert galaxies - solid upside-down triangles; broad-line AGNslarge, solid triangles; CS sources and our unclassified $\mathrm{z}=2.2032$ source [see §2.4] - solid circles; photometric redshifts-open squares; unidentified sources-open leftward-pointing triangles at $z<0$ ). Sources with radio fluxes greater than $2500 \mu \mathrm{Jy}$ are shown at that flux. (b) Redshift distribution for the radio sample with spectroscopic or photometric redshifts, divided into five flux bins. Radio flux ranges in microJanskys are given for each bin. Each tickmark on the y-axis represents five sources.

This invariance in both the optical apparent magnitudes and the redshifts of the host galaxies is a striking result. In Figure 13, we plot rest-frame $\mathrm{AB} 4500 \AA-8500 \AA$ color versus (a) redshift and (b) $1.4 \mathrm{GHz}$ flux for the spectroscopically (solid symbols) and photometrically (open squares) identified radio sources. Again, our uniform and complete wavelength coverage for all of the radio sources means that we can just interpolate between our measurements to obtain the rest-frame colors. We only use redshifts up to $z=1.2$ so that our interpolation remains valid. The solid and dotted lines show the rest-frame AB $4500 \AA$ 
- $8500 \AA$ A color of an elliptical and irregular galaxy, respectively, using Coleman et al. (1980). In both plots, there is very little color variation with redshift. The radio sources are clearly drawn from the same host galaxy population, and the host galaxy properties are not evolving much with redshift.
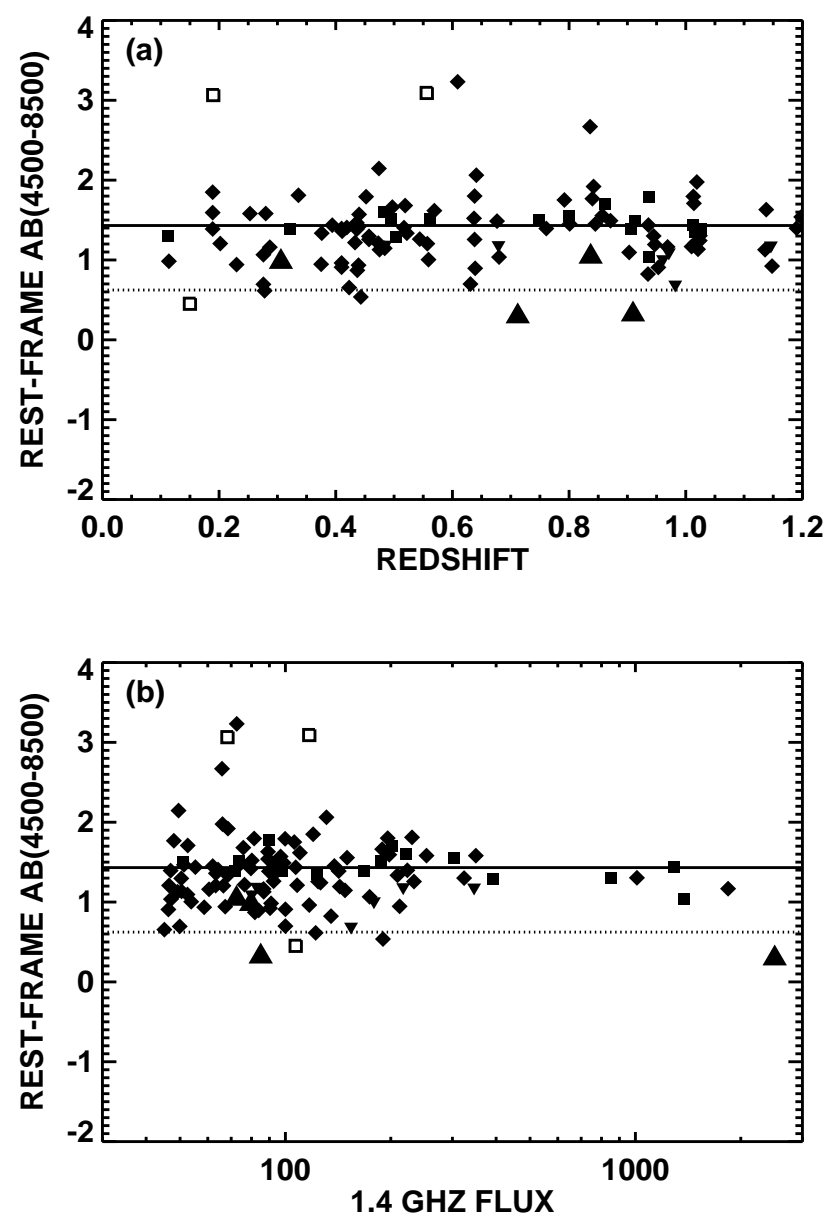

FIG. 13.- Rest-frame AB $4500 \AA-8500 \AA$ color vs. (a) redshift and (b) $1.4 \mathrm{GHz}$ flux for the $z<1.2$ spectroscopically (absorberssolid squares; star formers-solid diamonds; Seyfert galaxies-solid upside-down triangles; broad-line AGNs - large, solid triangles) and photometrically (open squares) identified radio sample. The solid (dotted) line shows the rest-frame color of an elliptical (irregular) galaxy from Coleman et al. (1980).

In Figure 14a, we plot the absolute rest-frame $R$ magnitude, $M_{R}$, versus redshift for the spectroscopically (solid symbols) and photometrically (open squares) identified radio sources, interpolating between our measurements to obtain rest-frame $\mathrm{AB} 6500 \AA$, and restricting to $z<1.6$ so that our interpolation remains valid. The approximate effect of our $R \sim 24$ spectroscopic selection limit on $M_{R}$ is shown by the dotted curve. The median absolute magnitude at $z<0.4$ is $M_{R}=-21.4$, while that between $z=0.4$ and $z=0.8$ is $M_{R}=-21.5$, so there may be a weak evolution to brighter magnitudes at higher redshift. However, this evolution is small, particularly when passive evolution of the galaxies is taken into account. At no redshift do the host galaxies become much brighter than $M_{R}=-23$. Thus, we see a narrow range in $M_{R}$ with redshift (dashed lines), which suggests that the radio sources are chosen from approximately $L^{*}$ optical galaxies at all redshifts. At a very crude level, the radio hosts are standard candles in their optical properties.
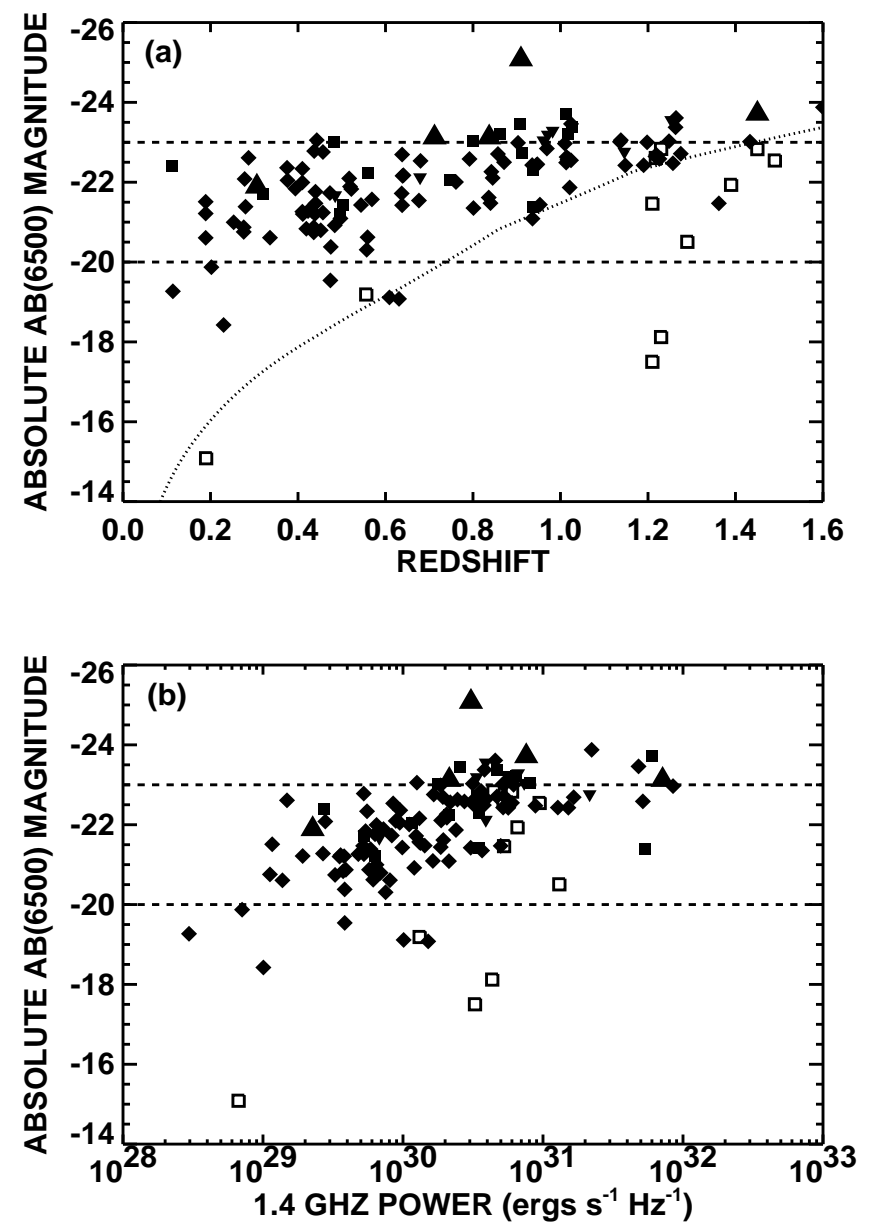

FIG. 14.- Absolute AB 6500 Å magnitude versus (a) redshift and (b) $1.4 \mathrm{GHz}$ power for the $z<1.6$ spectroscopically (absorberssolid squares; star formers-solid diamonds; Seyfert galaxies-solid upside-down triangles; broad-line AGNs - large, solid triangles) and photometrically (open squares) identified radio sample. Dashed lines show the approximate range in absolute magnitude, from -20 to -23 , where the majority of the radio sources lie. Dotted curve in (a) illustrates the effect of an $R \sim 24$ spectroscopic incompleteness limit on the absolute magnitudes.

In Figure 14b, we plot $M_{R}$ versus $1.4 \mathrm{GHz}$ power. Even though the galaxy hosts have similar optical luminosities at all redshifts, we can see from the figure that they have a wide range of radio powers (see also Cirasuolo et al. 2003). Thus, the radio powers of the host galaxies must be rising dramatically with increasing redshift, while the optical properties of the host galaxies are not changing.

We can see this dramatic evolution in Figure 15, which shows the spectroscopically (solid symbols) or photometrically (open squares) identified radio sample in a redshift versus 1.4 GHz power plot. The vertical dashed and solid lines show the radio powers (see $\S 9$ for how we determined these) corresponding to a luminous infrared galaxy (LIRG; $\left.10^{11} L_{\odot} \leq L_{F I R}<10^{12} L_{\odot}\right)$ and an ultraluminous infrared galaxy (ULIRG; $L_{F I R} \geq 10^{12} L_{\odot}$ ), respectively. All of the ULIRGs in the $1.4 \mathrm{GHz}$ sample to a completeness limit of $60 \mu \mathrm{Jy}$ (dotted curve) should be detected to $z \sim 1.3$. 
Interestingly, we see from Figure 15 that only a narrow range of luminosities is observed at each redshift and that very high-luminosity sources are primarily found at high redshifts $(z \gtrsim 1)$. We therefore conclude that similar optical galaxies (see Fig. 14a) are hosting more powerful radio galaxies at higher redshifts. (See Cowie et al. 2004a for a quantitative description of the evolution of the $1.4 \mathrm{GHz}$ luminosity function.)

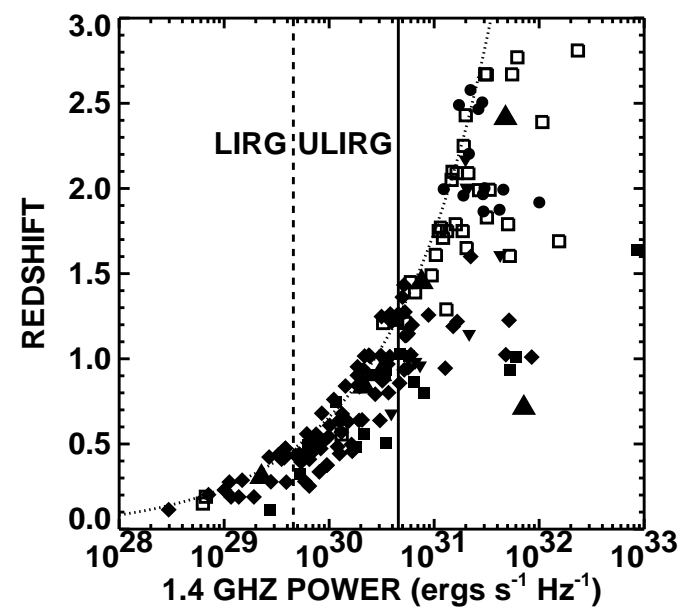

FIG. 15.- Redshift vs. $1.4 \mathrm{GHz}$ luminosity for the spectroscopically (absorbers-solid squares; star formers-solid diamonds; Seyfert galaxies-solid upside-down triangles; broad-line AGNslarge, solid triangles; CS sources and our unclassified $z=2.2032$ source [see §2.4]-solid circles) and photometrically (open squares) identified radio sample. The dashed and solid vertical lines show the equivalent radio powers of a LIRG and a ULIRG, respectively. The dotted curve shows the radio powers corresponding to the $60 \mu \mathrm{Jy}$ completeness limit of the radio sample.

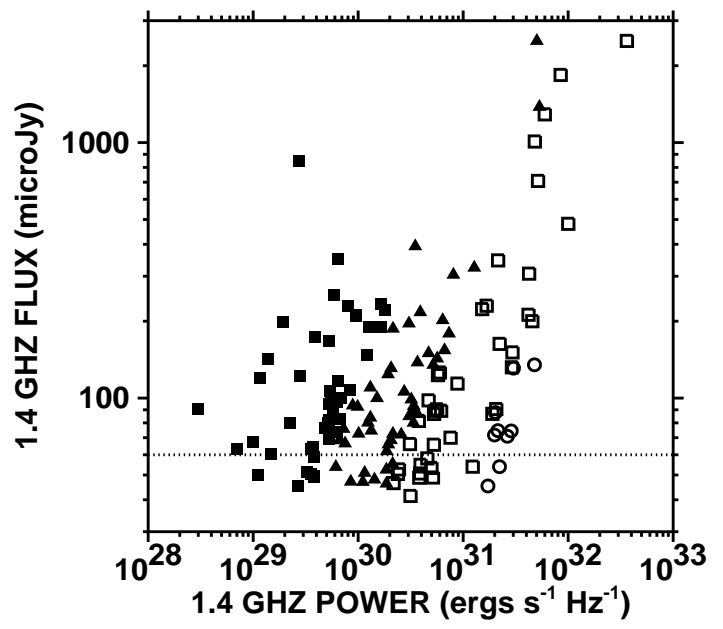

FIG. 16.- $1.4 \mathrm{GHz}$ flux versus $1.4 \mathrm{GHz}$ power for the spectroscopically identified radio sample, including the CS sources $(z<0.5-$ solid squares; $0.5 \leq z<1$-solid triangles; $1 \leq z<2$-open squares; $z \geq 2$-open circles). The dotted line shows the $60 \mu \mathrm{Jy}$ completeness limit. Sources with radio fluxes greater than $2500 \mu \mathrm{Jy}$ are shown at that flux.

We expand on this result in Figure 16, where we show $1.4 \mathrm{GHz}$ flux versus $1.4 \mathrm{GHz}$ power. Here we denote the different redshift intervals by different symbols $(z<0.5$, solid squares; $0.5 \leq z<1$, solid triangles; $1 \leq z<2$, open squares; $z \geq 2$, open circles). We see a series of increasing luminosity slices with increasing redshift. The reason for this effect was first suggested by Condon (1989) based on the radio source counts. At low redshifts $(z \lesssim 1)$, the radio surveys are dominated by the most luminous sources in each redshift shell because the radio population is experiencing such a dramatic luminosity evolution. This is unlike any other wavelength survey, where sources are generally picked up throughout the whole volume distribution. However, at higher redshifts, the evolution slows, and a wider range of luminosities is observed.

Figure 16 contradicts the suggestion by Ciliegi et al. (2005) that most of the faintest radio sources may be associated with relatively low radio luminosity sources at relatively modest redshifts rather than with high radio luminosity AGNs at high redshifts. In fact, the faintest radio sources have a wide range of radio powers and redshifts, with the higher radio powers corresponding to the higher redshifts.

\section{UPPER LIMITS ON HIGHLY-OBSCURED AGNS}

An important goal of the present work is to determine an upper limit on the number of AGNs with quasar-like bolometric luminosities $\left(\sim 4 \times 10^{45} \mathrm{ergs} \mathrm{s}^{-1}\right.$; see below $)$ that could be highly obscured - such that the light emerges in the FIR - and hence not be detected in current deep Xray surveys. This determination will be an upper limit, since for any of the radio sources, it is likely that much of the FIR light will be due to star formation rather than to AGN activity. Moreover, such a determination will contain radio-loud sources, where the FIR-radio correlation substantially overestimates the FIR.

We calculate total FIR luminosities for the radio sources, assuming that the FIR-radio correlation for star formers and radio-quiet AGNs holds at high redshifts, and assuming that the sources are well described by this correlation. Following Barger et al. (2001), we use the FIR-radio correlation given in Sanders \& Mirabel (1996) with $q=2.35$ that they find holds for sources covering several orders of magnitude in FIR luminosity. Then $f_{F I R}=8.4 \times 10^{14} f_{1.4}^{r f}$, where $f_{1.4}^{r f}$ is the rest-frame flux at $1.4 \mathrm{GHz}$. Assuming a synchrotron spectrum with a spectral index of 0.8 (Yun et al. 2001), we can calculate the total FIR luminosity from the observed $1.4 \mathrm{GHz}$ flux using

$$
L_{F I R}=4 \pi d_{L}^{2}\left(8.4 \times 10^{14}\right) f_{1.4}(1+z)^{-0.2},
$$

where $d_{L}$ is the luminosity distance in $\mathrm{cm}$ and $f_{1.4}$ has units of ergs $\mathrm{cm}^{-2} \mathrm{~s}^{-1} \mathrm{~Hz}^{-1}$.

Of particular interest for our analysis are the sources that do not also have high $\left(\geq 10^{42} \mathrm{ergs} \mathrm{s}^{-1}\right) \mathrm{X}$-ray luminosities. Quasars are typically defined as having $2-8 \mathrm{keV}$ X-ray luminosities $\geq 10^{44} \mathrm{ergs} \mathrm{s}^{-1}$, which, with a factor of 35 bolometric correction (e.g., Elvis et al. 1994; Kuraszkiewicz et al. 2003), would roughly correspond to ULIRG luminosities. Thus, we consider any ULIRGs that do not have high X-ray luminosities to be our primary candidates to contain highly-obscured AGNs.

In Figure 17, we plot redshift versus total FIR luminosity for the sources with ULIRG (vertical line) luminosities $L_{F I R} \geq 4 \times 10^{45} \mathrm{ergs} \mathrm{s}^{-1}$. We denote sources with spectroscopic redshifts by solid symbols and sources with photometric redshifts by open squares. We circle the sources that have either hard $(2-8 \mathrm{keV})$ or soft $(0.5-2 \mathrm{keV}) \mathrm{X}$ ray luminosities $\geq 10^{42} \mathrm{ergs} \mathrm{s}^{-1}$. The dotted curve shows the $60 \mu \mathrm{Jy}$ completeness limit for the radio observations. 
The dashed horizontal line shows an adopted upper redshift cut-off of $z=2$, which is a compromise to keep our ULIRG selection as uniform as possible (since the radio completeness limit begins to cut out the lower luminosity ULIRGs at $z \gtrsim 1.3$ ), while still retaining a statistically significant sample of sources.

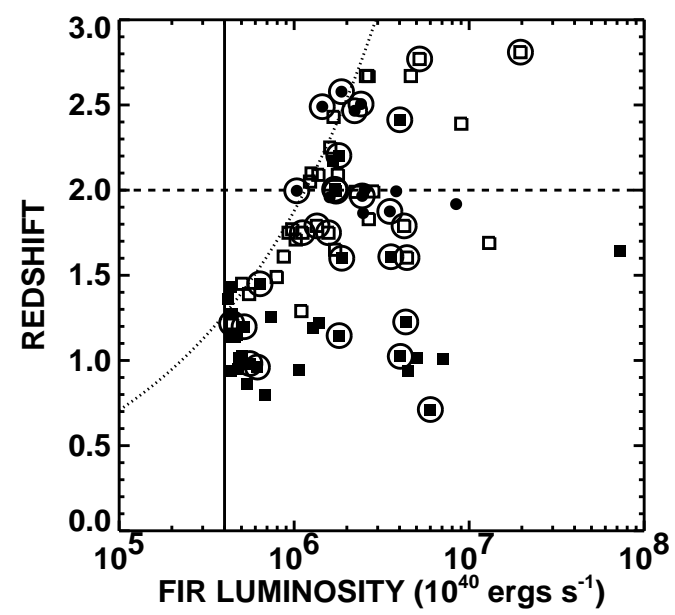

FIG. 17.- Redshift vs. total FIR luminosity for the radio sample with ULIRG luminosities. Solid squares and solid circles (the latter are CS sources) denote spectroscopic redshifts, and open squares denote photometric redshifts. Large, open circles denote sources

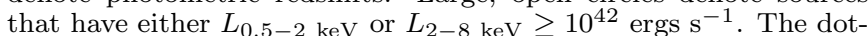
ted curve shows the $60 \mu \mathrm{Jy}$ completeness limit of the radio sample. The solid vertical line shows the minimum luminosity of a ULIRG. The dashed horizontal line shows an upper redshift cut-off of $z=2$ to provide as uniform a selection of ULIRGs as possible, while still retaining a statistically significant sample.

In Figure 18a, we show histograms of the logarithmic total FIR luminosities of the $z \leq 2$ radio sources with either spectroscopic or photometric redshifts and ULIRG luminosities divided into two categories: high $\mathrm{X}$-ray luminosity sources (either $L_{0.5-2 \mathrm{keV}}$ or $L_{2-8} \mathrm{keV} \geq 10^{42} \mathrm{ergs} \mathrm{s}^{-1}$ ) and low X-ray luminosity sources (both $L_{0.5-2} \mathrm{keV}$ and $\left.L_{2-8} \mathrm{keV}<10^{42} \mathrm{ergs} \mathrm{s}^{-1}\right)$. There are 38 sources in the low $\mathrm{X}$-ray luminosity histogram and 20 sources in the high $\mathrm{X}$ ray luminosity histogram. Excluding the bi-lobal source (the source with the highest FIR luminosity estimate in Fig. 18, which is likely a substantial overestimate for this radio-loud AGN) from being considered a candidate to contain a highly-obscured AGN, we find a maximum ratio of highly-obscured AGN candidates to high-luminosity Xray sources of 1.9 for radio sources in the quasar class. We stress again that this is an upper limit, since it includes star-forming ULIRGs as well as radio-loud AGNs, where the FIR-radio correlation is overestimating the FIR luminosity. Even if all 17 sources without photometric redshifts (11 of which do not have any obvious counterparts) were assumed to have ULIRG luminosities, to lie at $z \leq 2$, and not to be X-ray luminous, the ratio would only increase to 2.7 .

To show the classes of sources that are comprising the low X-ray luminosity population, in Figure 18b, we divide the population into absorbers, star formers, and "other". The latter category includes spectroscopically unclassified sources and sources with only photometric redshifts. In principle, we should be able to exclude the absorbers, which are presumably radio-loud AGNs in the centers of elliptical galaxies, from being considered candidates to con- tain highly-obscured AGNs. However, in practice, apart from the bi-lobal source, we leave the absorbers in because of the difficulties in distinguishing between radio-loud and radio-quiet AGNs, as discussed in $\S 6.1$.
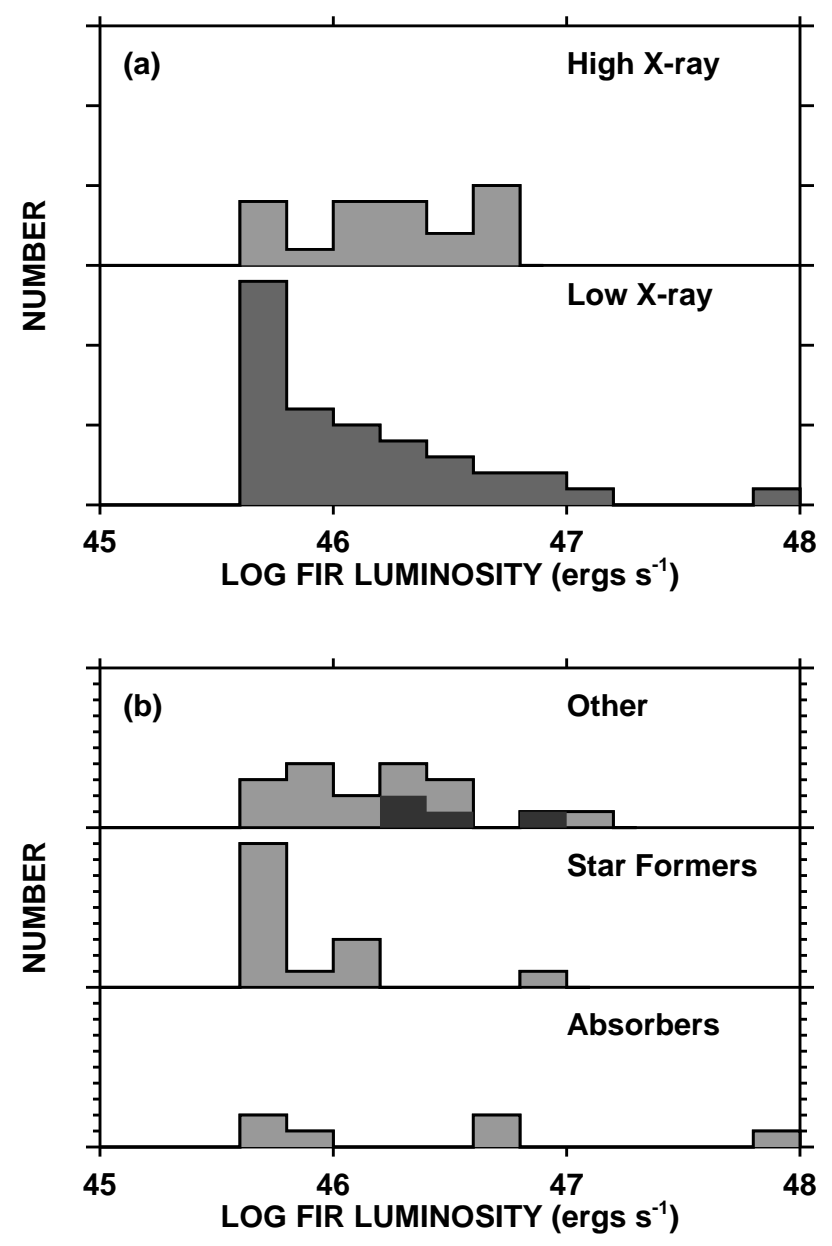

FIG. 18.- (a) Histograms of logarithmic FIR luminosity for the $L_{F I R} \geq 4 \times 10^{45} \mathrm{ergs} \mathrm{s}^{-1}$ and $z \leq 2$ radio sample with spectroscopic or photometric redshifts, divided into two X-ray luminosity bins: high X-ray (either $L_{0.5-2 \mathrm{keV}}$ or $L_{2-8 \mathrm{keV}} \geq 10^{42} \mathrm{ergs} \mathrm{s}^{-1}$ ) and low X-ray (both $L_{0.5-2} \mathrm{keV}$ and $L_{2-8} \mathrm{keV}<10^{42} \mathrm{ergs} \mathrm{s}^{-1}$ ). Each tickmark on the y-axis represents five sources. (b) Histograms of the low X-ray luminosities from (a) divided into three categories: absorbers, star formers, and "other" (spectroscopically unclassified sources and sources with only photometric redshifts). The CS sources are denoted by dark shading. Each tickmark on the y-axis represents one source.

In Figure 19, we show in two redshift bins the number densities of radio sources with either spectroscopic or photometric redshifts and $L_{F I R} \geq 4 \times 10^{45} \mathrm{ergs} \mathrm{s}^{-1}$, divided according to X-ray luminosity. At these high FIR luminosities, the ratio of highly-obscured AGN candidates to high X-ray luminosity sources does not appear to be changing much with redshift, with values of 2 and 1.8 for the lower and higher redshift bins.

We can get a rough check on whether the upper bound on the ratio continues to stay about the same at lower FIR luminosities, and hence lower redshifts, by redoing our analysis for LIRGs. Of course, there is much more contamination at these lower luminosities by star formation, since M82-type sources will push into the sample and will not show up as luminous X-ray sources. In Fig- 
ure 20, we again plot redshift versus total FIR luminosity, this time for the radio sources with LIRG luminosities $\left(4 \times 10^{44} \leq L_{F I R}<4 \times 10^{45} \mathrm{ergs} \mathrm{s}^{-1}\right.$; vertical lines $)$. The dashed horizontal line shows an adopted upper redshift cut-off of $z=0.7$. The ratio of highly-obscured AGN candidates to high X-ray luminosity sources for the LIRG population with $0.2<z \leq 0.7$ comes out to be 5.4 , which can be taken as an extreme upper limit.

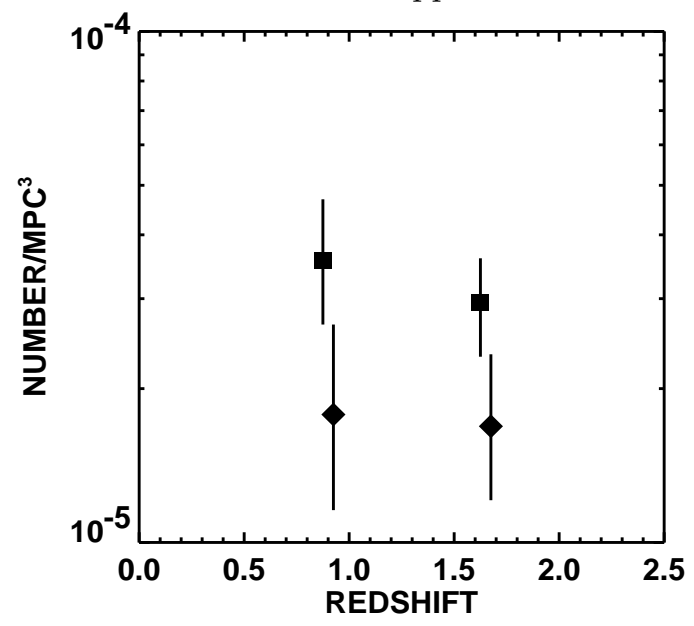

FIG. 19. - Number densities in two redshift bins $(0.5<z<1.25$ and $1.25<z \leq 2$ ) of radio sources with either spectroscopic or photometric redshifts and $L_{F I R} \geq 4 \times 10^{45} \mathrm{ergs} \mathrm{s}^{-1}$, divided according to X-ray luminosity: diamonds denote sources with either $L_{0.5-2} \mathrm{keV}$ or $L_{2-8 \mathrm{keV}} \geq 10^{42} \mathrm{ergs} \mathrm{s}^{-1}$, and squares denote sources with both soft and hard X-ray luminosities $<10^{42} \mathrm{ergs} \mathrm{s}^{-1}$. Squares are slightly offset for clarity. Poissonian $1 \sigma$ uncertainties are based on the number of sources in each redshift interval.

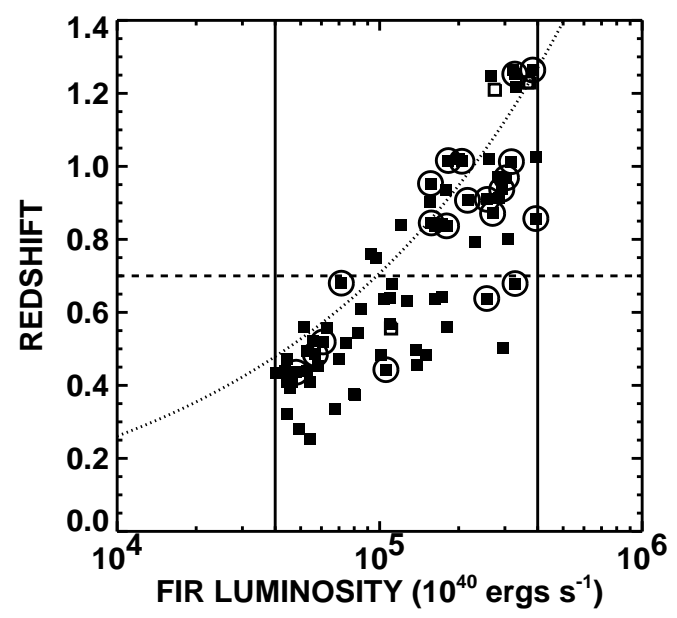

FIG. 20.- Redshift vs. total FIR luminosity for the radio sample with LIRG luminosities. Solid squares and solid circles (the latter are CS sources) denote spectroscopic redshifts, and open squares denote photometric redshifts. Large, open circles denote sources that

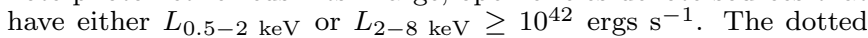
curve shows the $60 \mu \mathrm{Jy}$ completeness limit of the radio sample. The two solid vertical lines show the minimum luminosities of a LIRG and a ULIRG. The dashed horizontal line shows an upper redshift cut-off of $z=0.7$ to provide as uniform a selection of LIRGs as possible, while still retaining a statistically significant sample.

Models of the XRB generally require a population of near-Compton-thick sources $\left(N_{H} \sim 10^{23-24} \mathrm{~cm}^{-2}\right)$. Typical estimates are, on average, for $2-3$ times as many such sources as X-ray detected sources (e.g., Fabian \& Worsley 2004; Treister \& Urry 2005). However, such obscured sources may preferentially lie at luminosities lower than quasar luminosities (e.g., Ballantyne et al. 2006). Thus, our upper limit of 1.9 for the ratio of quasar-luminosity but not X-ray-luminous sources to quasar-luminosity, Xray-luminous sources may be consistent with these results.

If we assume that a roughly factor of two ratio of highly-obscured AGN candidates to high X-ray luminosity sources applies to the quasar luminosity population and shows no redshift effect, then we estimate that the accreted supermassive black hole mass density determined by Barger et al. (2005) for broad-line AGNs (their Eq. 9) will increase to $3.6 \times 10^{5} M_{\odot} \mathrm{Mpc}^{-3}$. Although this is still within $2 \sigma$ of the local supermassive black hole mass density found by $\mathrm{Yu} \&$ Tremaine $(2002)$ of $(2.9 \pm 0.5) \times$ $10^{5} M_{\odot} \mathrm{Mpc}^{-3}$ for $h=0.7$, it does not leave much room for the obscured accretion from the optically narrow AGNs. Applying the same factor of two corrections to Equations 6 and 7 from Barger et al. (2005), which give the accreted supermassive black hole mass density for all spectral types assuming bolometric corrections, respectively, of 85 and 35 for the optically narrow AGNs, would increase these numbers to $12 \times 10^{5}$ and $6.3 \times 10^{5} M_{\odot} \mathrm{Mpc}^{-3}$. This could be viewed as a consistency check that there are not that many luminous obscured AGNs.

\section{CONTRIBUTIONS TO THE X-RAY LIGHT}

We would now like to understand what fraction of the $\mathrm{X}$-ray light is coming from the different $\mathrm{X}$-ray and radio populations, especially from the radio-identified ULIRG population. To study this, we performed source-stacking analyses on the CDF-N 2 Ms exposure, restricting to a $9.5^{\prime}$ radius circle rather than the $10^{\prime}$ radius circle used elsewhere in this paper, since a few of the radio sources in the $10^{\prime}$ radius circle lie just outside the $\mathrm{X}$-ray image.

For each sample, we first measured the X-ray fluxes of all of the sources in each of four passbands, $0.5-1,1-2$, $2-4$, and $4-8 \mathrm{keV}$, using the images and exposure maps given in Alexander et al. (2003). For the X-ray sample, we measured the fluxes of all of the sources in the Alexander et al. (2003) catalog that lie in the $9.5^{\prime}$ radius region. (Note that Worsley et al. 2005 found an increase in total resolved flux of about $2-5 \%$ in the $>2 \mathrm{keV}$ bands when they applied the systematic flux correction determined by Bauer et al. (2004) to account for Eddington bias and some additional aperture/photometry effects that were not considered in Alexander et al. 2003, but we have not applied that correction here.)

To calculate the contribution to the extragalactic background light (EBL) from a given sample, we summed the fluxes in the $9.5^{\prime}$ radius circle and divided by the corresponding area. We did not exclude any sources from the summations, even if a source were not significantly detected in a particular band or had a measured negative flux in the band. This simple averaging is not optimal, since the errors in the X-ray fluxes are smaller at smaller off-axis angles, but it avoids weighting the smaller central region more highly.

To estimate the uncertainties on our surface brightness measurements, we first generated a grid of 60,000 points in the $9.5^{\prime}$ radius area that are separated from each other by more than $4^{\prime \prime}$ (the aperture diameter used to measure the counts in the X-ray sources) and are located more than $6^{\prime \prime}$ away from any known X-ray source. We next measured 
the fluxes in each of our four X-ray passbands at these 60,000 positions. This serves as our base file. Then, for each sample for which we want to determine the uncertainties, we randomly generate a number (which matches the number of sources in the sample) of integers between 1 and 60,000 and use the fluxes corresponding to those positions in our base file. We do this 1000 times, which gives us 1000 flux values for each of the four passbands. We then order those values from the most negative to the most positive and use the $68 \%$ confidence interval to determine our uncertainties. (Most of our uncertainties are much smaller than the data points.)

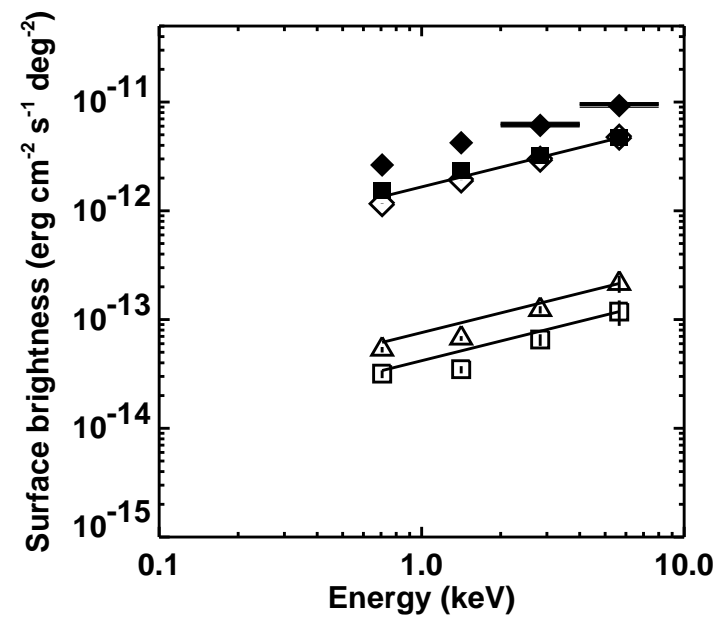

FIG. 21. - X-ray surface brightness vs. energy measured within a 9.5' radius circle for the Alexander et al. (2003) X-ray sample (solid diamonds), for the X-ray sample without radio counterparts (open diamonds), for the present radio sample (solid squares), for the radio sample without X-ray-luminous counterparts (these sources

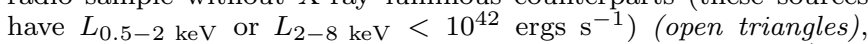
and for the radio sample without X-ray counterparts at all (open squares). The uncertainties are $68 \%$ confidence intervals, as described in the text. For comparison purposes, the diagonal solid lines show the 1.4 photon index of the XRB normalized to the highest energy bins of the X-ray surface brightness measurements for the radio sample, for the non-X-ray-luminous radio sample, and for the X-ray-undetected radio sample. The horizontal solid lines show the 2-10 keV XRB measurement of Revnivtsev et al. (2005) converted into narrower energy bands by adopting a photon index of 1.4. The thickness of the lines represents the uncertainties on their measurement.

In Figure 21, we show the EBL from the X-ray sources in the Alexander et al. (2003) catalog with solid diamonds. Coincidentally, they match very well the $2-10 \mathrm{keV}$ XRB measurement made by Revnivtsev et al. (2005) from a reanalysis of the HEAO1/A2 data (thick horizontal lines; the thickness denotes the uncertainty range on their measurement). Note that we converted the Revnivtsev et al. (2005) $2-10 \mathrm{keV}$ measurement to $2-4 \mathrm{keV}, 4-8 \mathrm{keV}$, and $8-10 \mathrm{keV}$ by adopting a photon index of 1.4 . We chose this determination to compare with since the A2 or Cosmic X-ray Experiment instrument was especially designed for accurate measurements of the XRB over a very wide sky solid angle (e.g., Marshall et al. 1980; Boldt 1987; Gruber et al. 1999), and the design of its detectors allows the internal instrumental background to be separated from the XRB with almost absolute accuracy (Rothschild et al. 1979; Boldt 1987).

However, for this work, we are not interested in accurately determining the resolved fraction of the XRB.
Such an analysis would require integrating the known $\log N-\log S$ distribution to take into account the rare, bright sources that are not sampled in this deep pencilbeam survey, extrapolating the number counts to take into account the sources with fainter fluxes, and deciding which of the XRB measurements to compare with. Indeed, this was done by Moretti et al. (2003), and there is very good agreement between their result and the Revnivtsev et al. (2005) measurement. Rather, it is sufficient for us to measure the X-ray surface brightnesses from the known Xray sources in the CDF-N and see that they are roughly consistent with the HEAO1/A2 XRB measurement. In other words, we are not concerned with uncertainties at the $10-20 \%$ level. Our aim is just to see roughly what fraction of the X-ray light comes from the various populations.

We begin by considering only the Alexander et al. (2003) $\mathrm{X}$-ray sources that are not radio sources in the Richards (2000) catalog. In Figure 21, we show the computed Xray surface brightnesses from these sources with open diamonds. We find that they contribute $50 \pm 0.7 \%$ of the $\mathrm{X}$-ray light in the $4-8 \mathrm{keV}$ band.

Next, we examine how much the total radio sample contributes to the X-ray light. In Figure 21, we show our computed X-ray surface brightnesses for this sample with solid squares. We see that the radio sample also contributes $50 \pm 0.5 \%$ of the X-ray light in the $4-8 \mathrm{keV}$ band. In other words, radio sources to the Richards (2000) $1.4 \mathrm{GHz}$ flux limit of $40 \mu \mathrm{Jy}$ are markers for half of the X-ray light at these high energies. This contribution is the same as the contribution from the X-ray sources that are not detected at these radio fluxes.

However, most of the light contributed by the radio sample comes from the X-ray-luminous radio sources. To show this, we next measure the $\mathrm{X}$-ray surface brightnesses of the sample of radio sources without X-rayluminous counterparts (these sources have $L_{0.5-2} \mathrm{keV}$ or $L_{2-8 \mathrm{keV}}<10^{42} \mathrm{ergs} \mathrm{s}^{-1}$; hereafter, non-X-ray-luminous) in the Alexander et al. (2003) catalog (open triangles), as well as of the sample of radio sources without X-ray counterparts at all (hereafter, X-ray-undetected) (open squares).

We find the contributions to the $4-8 \mathrm{keV}$ light from the non-X-ray-luminous population to be $2.3 \pm 0.4 \%$ and from the X-ray-undetected population to be $1.2 \pm 0.3 \%$. Thus, although some of the radio sources are contributing additional X-ray light that is not already in the X-ray sample, such contributions are very small. This immediately tells us that the current radio source population cannot account for the background light that has been suggested may be missing at these energies. Indeed, the percentages are about a factor of 5 lower than those predicted for $N_{H} \sim 10^{24} \mathrm{~cm}^{-2}$ sources at these energies in typical XRB synthesis models (e.g., Comastri, Gilli, \& Hasinger 2006).

However, the inverse statement that some of these non$\mathrm{X}$-ray-luminous or X-ray-undetected radio sources contain highly-obscured AGNs is true. We can see this by comparing the shapes of the X-ray surface brightness measurements for the various samples with the shape of the XRB. To make it easier to do the comparisons, in Figure 21, we normalize the 1.4 photon index of the XRB to the surface brightnesses in the highest energy bin for all but the X-ray sample and the X-ray sample without 
radio counterparts (to avoid cluttering up the figure too much). We can see that the shape of the surface brightness measurements for the radio sample is slightly softer than that of the XRB, while the shape of the surface brightness measurements for the X-ray sample without radio counterparts is slightly harder than the XRB. However, the shapes of the surface brightness measurements for the non$\mathrm{X}$-ray-luminous radio sample and the X-ray-undetected radio sample both appear much harder than that of the $\mathrm{XRB}$. This result suggests that these two populations are consistent with containing highly-obscured AGNs.
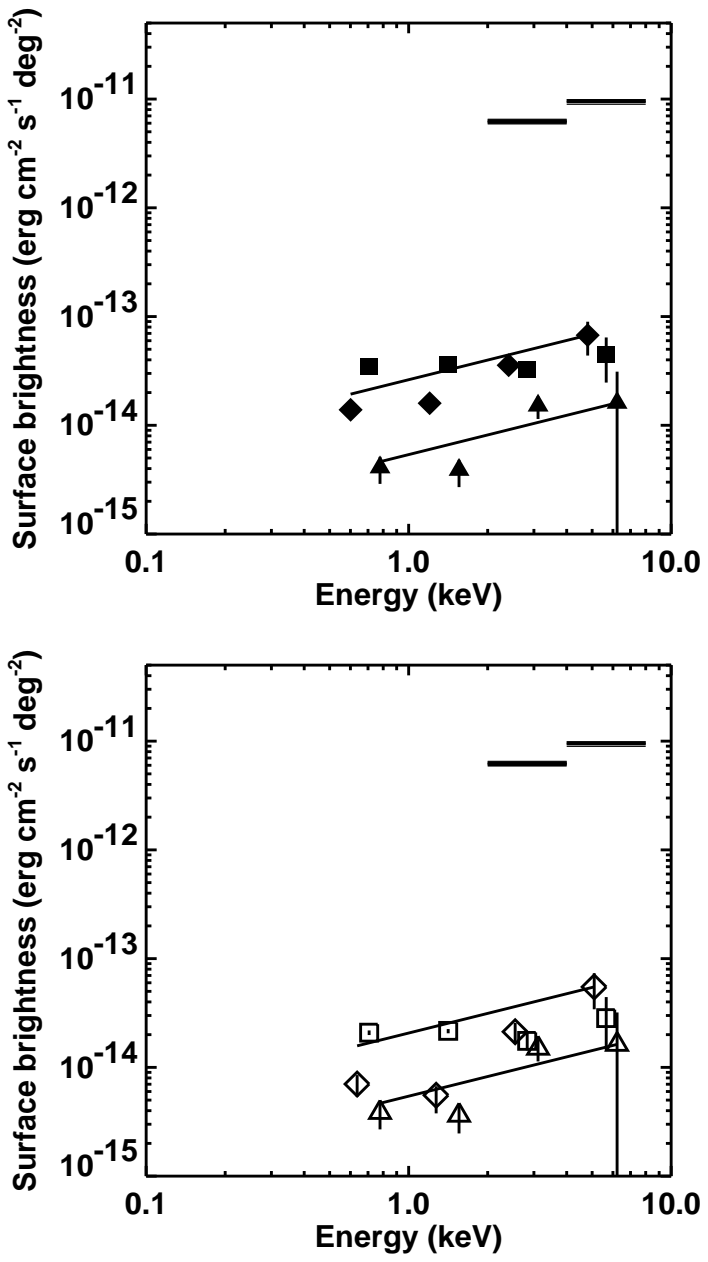

FIG. 22. - X-ray surface brightness vs. energy measured within a $9.5^{\prime}$ radius circle for three redshift intervals: $0<z \leq 0.5$ (squares), $0.5<z \leq 1.5$ (diamonds), and $1.5<z \leq 3$ (triangles) (a) for the radio sample without X-ray-luminous counterparts (these sources have $L_{0.5-2 \mathrm{keV}}$ or $L_{2-8} \mathrm{keV}<10^{42} \mathrm{ergs} \mathrm{s}^{-1}$ ) in the Alexander et al. (2003) catalog (solid symbols) and (b) for the radio sample without X-ray counterparts at all (open symbols). Small offsets have been applied in the $x$-direction to the diamonds and to the triangles for clarity. The uncertainties are $68 \%$ confidence intervals, as described in the text. For comparison purposes, the diagonal solid lines show the 1.4 photon index of the XRB normalized to the highest energy bins of the $0.5<z \leq 1.5$ and $1.5<z \leq 3$ samples. The horizontal solid lines show the $2-10 \mathrm{keV}$ XRB measurement of Revnivtsev et al. (2005) converted into narrower energy bands by adopting a photon index of 1.4. The thickness of the lines represents the uncertainties on their measurement.

One might wonder how the shapes of the X-ray surface brightness measurements for the non-X-ray-luminous radio sample and for the $\mathrm{X}$-ray-undetected radio sample vary with redshift. We show this in Figures 22a and b for the redshift intervals $z=0-0.5$ (squares), $0.5-1.5$ (diamonds), and $1.5-3$ (triangles). The solid symbols in Figure 22a denote the non-X-ray-luminous radio sample, and the open symbols in Figure $22 \mathrm{~b}$ denote the X-rayundetected radio sample. Small offsets have been applied in the $x$-direction to the diamonds and to the triangles for clarity. The uncertainties are again the $68 \%$ confidence intervals. We normalize the highest energy bins of the $z=0.5-1.5$ and $z=1.5-3$ samples to the 1.4 photon index of the XRB for comparison. In both figures, we can see that the lowest redshift interval sample (squares) is quite soft due to the contributions from star-forming galaxies, while the higher redshift interval samples are hard due to the obscured nature of the sources.

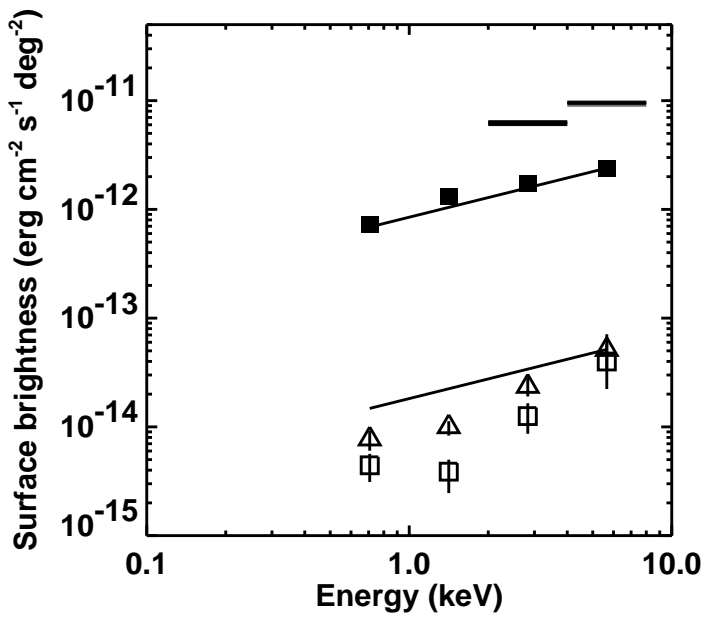

FIG. 23.- X-ray surface brightness vs. energy measured within a $9.5^{\prime}$ radius circle for the $z \leq 2$ radio-identified ULIRG sample with X-ray-luminous $\left(L_{0.5-2} \mathrm{keV}\right.$ or $\left.L_{2-8 \mathrm{keV}} \geq 10^{42} \mathrm{ergs} \mathrm{s}^{-1}\right)$ counterparts in the Alexander et al. (2003) catalog (solid squares), without X-ray luminous counterparts (open triangles), and without X-ray counterparts at all (open squares). For comparison purposes, the diagonal solid lines show the 1.4 photon index of the XRB normalized to the highest energy bins of the X-ray-luminous and non-X-ray-luminous samples. The horizontal solid lines show the $2-10 \mathrm{keV}$ XRB measurement of Revnivtsev et al. (2005) converted into narrower energy bands by adopting a photon index of 1.4. The thickness of the lines represents the uncertainties on their measurement.

Since in $\S 9$ we focused on the $z \leq 2$ radio-identified ULIRG sample, we now repeat our source-stacking analyses using only these data. Our results are shown in Figure 23. We use solid squares to denote the X-ray surface brightnesses measured for the $z \leq 2$ ULIRG sample with

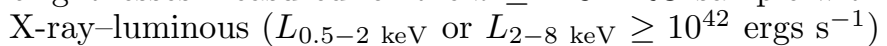
counterparts in the Alexander et al. (2003) catalog. These sources contribute $25 \pm 0.1 \%$ of the X-ray light in the $4-8 \mathrm{keV}$ band, which shows that sources with ULIRG radio powers are responsible for contributing a very substantial fraction of the XRB. We use open triangles to denote our measurements for the $z \leq 2$ non-X-ray-luminous ULIRGs. These contribute only $0.5 \pm 0.2 \%$ of the X-ray light in the $4-8 \mathrm{keV}$ band. Finally, we use open squares to denote our measurements for the $z \leq 2$ ULIRGs with no Xray detections at all in the Alexander et al. (2003) catalog. These contribute only $0.4 \pm 0.2 \%$ to the $4-8 \mathrm{keV}$ light. The uncertainties are again the $68 \%$ confidence intervals. We normalize the highest energy bins of the X-ray-luminous ULIRGs and the non-X-ray-luminous ULIRGs to the 1.4 photon index of the XRB for comparison. We see that the shape of the X-ray-luminous ULIRGs is quite well 
matched to the XRB, while the shapes of the non-X-rayluminous ULIRGs and the X-ray-undetected ULIRGs are considerably harder, rising steeply towards the higher energies. Again, this is consistent with these samples being highly obscured. However, even if we interpret them as such, their contributions to the $4-8 \mathrm{keV}$ light are very small, and they are unlikely to contribute substantially to the XRB at even higher energies.

\section{SUMMARY}

In this paper, we analyzed the nature and evolution of microJansky radio sources using a highly spectroscopically complete, deep VLA survey of the HDF-N region. We supplemented the spectroscopic identifications with photometric redshifts measured from the rest-frame ultraviolet to MIR spectral energy distributions. Our results are as follows.

- We found that the fraction of radio sources that can be optically spectroscopically identified is fairly independent of radio flux, with about $60-80 \%$ identified at all fluxes. We spectrally classified the galaxies into four spectral types: absorbers, star formers, Seyfert galaxies, and broad-line AGNs.

- We did not confirm the existence of an X-ray-radio correlation for star-forming galaxies in either the hard or soft X-ray bands. Previous claims of such a correlation appear to be the result of selection effects.

- We did not observe any correlation between $1.4 \mathrm{GHz}$ flux and $R$ magnitude, which helps to explain the uniformity of the spectroscopically identified fraction with radio flux. Previous claims of the existence of such a correlation were likely due to the much shallower optical observations that were used in those studies.

- We also did not observe any correlation between 1.4 GHz flux and redshift for the spectroscopically and photometrically identified sources. The redshift distribution is consistent with being invariant with radio flux, though we may be missing the higher redshift tail of the redshift distribution function due to optical spectroscopic bias.

- The observed invariance in the rest-frame colors and only small amount of change in the absolute magnitudes of the host galaxies suggests that radio observations sample the same type of host galaxies at all redshifts and that the host galaxy properties are not evolving much with redshift.

- There is dramatic evolution in the radio powers of the host galaxies with increasing redshift, and similar optical galaxies are hosting more powerful radio galaxies at higher redshifts. A quantitative description the evolution of the $1.4 \mathrm{GHz}$ luminosity function can be found in Cowie et al. (2004a).
- Assuming that the locally determined FIR-radio correlation holds at high redshifts, we estimated total FIR luminosities for the radio sources. We note that these will be overestimates in the case of radio-loud AGNs, but we conservatively did not try to remove the radio-loud AGNs from the sample, since we are only determining upper limits. For the radio sources with total FIR luminosities comparable to quasar-like bolometric luminosities, we obtained an upper limit of 1.9 for the ratio of candidate highly-obscured AGNs to X-ray-luminous sources.

- We measured the X-ray surface brightnesses for various X-ray and radio populations within a $9.5^{\prime}$ radius circle to determine their contributions to the X-ray light. For the known X-ray sources in the CDF-N, we found our measurements to be (coincidentally) consistent with the HEAO1/A2 XRB measurement made by Revnivtsev et al. (2005). We found that the Richards (2000) radio sample contributes half of the $4-8 \mathrm{keV}$ light, which is also how much the X-ray sources without radio counterparts contribute. However, most of the light from the radio sample comes from the X-ray-luminous radio sources. The non$\mathrm{X}$-ray-luminous and X-ray-undetected subsamples contribute only $2.3 \pm 0.4 \%$ and $1.2 \pm 0.3 \%$, respectively. This tells us that the current radio source population cannot account for the background light that one might think is missing at these energies. However, the shapes of the surface brightness measurements for these two samples both appear much harder than that of the XRB, which suggests that these two populations are consistent with containing highly-obscured AGNs. For the $z \leq 2$ radio-identified ULIRG sample, we found that the X-ray-luminous subsample contributes a very substantial one-quarter of the 4-8 keV light, while the non-X-ray-luminous and X-rayundetected subsamples each contribute less than a percent. Again, the shapes of the latter two subsamples are considerably harder than the XRB and hence are consistent with containing highly-obscured AGNs.

We thank the referee for helpful comments that improved the manuscript. We thank L. Silva for providing the template spectral energy distributions. This research has made use of the NASA/IPAC Extragalactic Database (NED) which is operated by the Jet Propulsion Laboratory, California Institute of Technology, under contract with the National Aeronautics and Space Administration. We gratefully acknowledge support from NSF grants AST 02-39425 (A. J. B.) and AST 04-07374 (L. L. C.), the University of Wisconsin Research Committee with funds granted by the Wisconsin Alumni Research Foundation, the Alfred P. Sloan Foundation, and the David and Lucile Packard Foundation (A. J. B.).

\section{REFERENCES}

Afonso, J., Georgakakis, A., Almeida, C., Hopkins, A. M., Cram, L. E., Mobasher, B., \& Sullivan, M. 2005, ApJ, 624, 135

Alexander, D. M., et al. 2003, AJ, 126, 539

Alonso-Herrero, A., et al. 2006, ApJ, 640, 167

Appleton, P. N., et al. 2004, ApJS, 154, 147

Ballantyne, D. R., Everett, J. E., \& Murray, N. 2006, ApJ, 639, 740

Barger, A. J., Cowie, L. L., Brandt, W. N., Capak, P., Garmire, G. P., Hornschemeier, A. E., Steffen, A. T., \& Wehner, E. H. 2002, AJ, 124,1839

Barger, A. J., Cowie, L. L., Mushotzky, R. F., \& Richards, E. A. 2001, AJ, 121, 662
Barger, A. J., Cowie, L. L., Mushotzky, R. F., Yang, Y., Wang, W.H., Steffen, A. T., \& Capak, P. 2005, AJ, 129, 578

Barger, A. J., Cowie, L. L., \& Richards, E. A. 2000, AJ, 119, 2092

Barger, A. J., et al. 2003, AJ, 126, 632

Bauer, F. E., Alexander, D. M., Brandt, W. N., Hornschemeier, A. E., Vignali, C., Garmire, G. P., \& Schneider, D. P. 2002, AJ, 124, 2351

Bauer, F. E., Alexander, D. M., Brandt, W. N., Schneider, D. P., Treister, E., Hornschemeier, A. E., \& Garmire, G. P. 2004, AJ, 128,2048

Boldt, E. 1987, Phys. Rep., 146, 215 
Brandt, W. N., et al. 2001, AJ, 122, 2810

Capak, P., et al. 2004, AJ, 127, 180

Chapman, S. C., Smail, I., Blain, A. W., \& Ivison, R. J. 2004, ApJ, 614,671

Chapman, S. C., Blain, A. W., Smail, I., \& Ivison, R. J. 2005, ApJ, 622,772

Ciliegi, P., et al. 2005, A\&A, 441, 879

Cirasuolo, M., Magliocchetti, M., Celotti, A., \& Danese, L. 2003, MNRAS, 341, 993

Cohen, J. G., et al. 2000, ApJ, 538, 29

Coleman, G. D., Wu, C.-C., \& Weedman, D. W. 1980, ApJS, 43, 393

Comastri, A. 2004, in Supermassive Black Holes in the Distant Universe, ed. A. J. Barger, (Dordrecht: Kluwer), 245

Comastri, A., Gilli, R., \& Hasinger, G. 2006, in Experimental Astronomy, in press (astro-ph/0604523)

Condon, J. J. 1989, ApJ, 338, 13

Condon, J. J. 1992, ARA\&A, 30, 575

Condon, J. J., Anderson, M. L., \& Helou, G. 1991, ApJ, 376, 95

Cowie, L. L., Barger, A. J., Fomalont, E. B., \& Capak, P. 2004a, ApJ, 603, L69

Cowie, L. L., Barger, A. J., Hu, E. M., Capak, P., \& Songaila, A. 2004b, AJ, 127, 3137

Cowie, L. L., Gardner, J. P., Hu, E. M., Songaila, A., Hodapp, K.-W., \& Wainscoat, R. J. 1994, ApJ, 434, 114

Dale, D. A., et al. 2005, ApJ, 633, 857

De Luca, A., \& Molendi, S. 2004, A\&A, 419, 837

Donley, J. L., Rieke, G. H., Rigby, J. R., \& Pérez-González, P. G. 2005, ApJ, 634, 169

Elbaz, D., et al. 2002, A\&A, 384, 848

Elvis, M., et al. 1994, ApJS, 95, 1

Faber, S. M., et al. 2003, Proc. SPIE, 4841, 1657

Franceschini, A., Braito, V., \& Fadda, D. 2002, MNRAS, 335, L51

Gandhi, P., \& Fabian, A. C. 2003, MNRAS, 339, 1095

Garrett, M. A. 2002, A\&A, 384, L19

Georgakakis, A., Mobasher, B., Cram, L., Hopkins, A., Lidman, C., \& Rowan-Robinson, M. 1999, MNRAS, 306, 708

Giavalisco, M., et al. 2004, ApJ, 600, L93

Gilfanov, M., Grimm, H.-J., \& Sunyaev, R. 2004, MNRAS, 347, L57

Gilli, R. 2004, Adv. Space Res., 34, 2470

Grimm, H.-J., Gilfanov, M., \& Sunyaev, R. 2003, MNRAS, 339, 793

Gruber, D. E., Matteson, J. L., Peterson, L. E., \& Jung, G. V. 1999, ApJ, 520, 124

Gruppioni, C., et al. 2003, MNRAS, 341, L1

Guainazzi, M., Matt, G., \& Perola, G. C. 2005, A\&A, 444, 119

Helou, G., Soifer, B. T., \& Rowan-Robinson, M. 1985, ApJ, 298, L7

Hickox, R. C., \& Markevitch, M. 2006, ApJ, 645, 95

Ivezić, Z., et al. 2002, AJ, 124, 2364

Kellermann, K. I., Sramek, R., Schmidt, M., Shaffer, D. B., \& Green, R. 1989, AJ, 98, 1195

Kuraszkiewicz, J. K., et al. 2003, ApJ, 590, 128

Lanzetta, K. M., Yahil, A., \& Fernandez-Soto, A. 1996, Nature, 381, 759

Loose, M., Farris, M. C., Garnett, J. D., Hall, D. N. B., \& Kozlowski, L. J. 2003, Proc. SPIE, 4850, 867

Machalski, J., \& Condon, J. J. 1999, ApJS, 123, 41

Maiolino, R., \& Rieke, G. H. 1995, ApJ, 454, 95
Maiolino, R., Salvati, M., Bassani, L., Dadina, M., della Ceca, R., Matt, G., Risaliti, G., \& Zamorani, G. 1998, A\&A, 338, 781

Marcillac, D., Elbaz, D., Chary, R. R., Dickinson, M., Galliano, F., \& Morrison, G. 2006, A\&A, 451, 57

Marshall, F., et al. 1980, ApJ, 235, 4

Martínez-Sansigre, A., Rawlings, S., Lacy, M., Fadda, D., Marleau, F. R., Simpson, C., Willott, C. J., \& Jarvis, M. J. 2005, Nature, 436,666

Monet, D. G., et al. 2003, AJ, 125, 984

Moran, E. C., Lehnert, M. D., \& Helfand, D. J. 1999, ApJ, 526, 649

Moretti, A., Campana, S., Lazzati, D., \& Tagliaferri, G. 2003, ApJ, 588,696

Norman, C., et al. 2004, ApJ, 607, 721

Oke, J. B., et al. 1995, PASP, 107, 375

Pérez-González, P. G., et al. 2005, ApJ, 630, 82

Polletta, M., et al. 2006, ApJ, 642, 673

Ranalli, P., Comastri, A., \& Setti, G. 2003, A\&A, 399, 39

Revnivtsev, M., Gilfanov, M., Jahoda, K., \& Sunyaev, R. 2005, A\&A, 444,381

Richards, E. A., Kellermann, K. I., Fomalont, E. B., Windhorst, R. A., \& Partridge, R. B. 1998, AJ, 116, 1039

Richards, E. A. 2000, ApJ, 533, 611

Risaliti, G., Maiolino, R., \& Salvati, M. 1999, ApJ, 522, 157

Roche, N. D., Lowenthal, J. D., \& Koo, D. C. 2002, MNRAS, 330, 307

Rothschild, R., et al. 1979, Space Sci. Instr., 4, 269

Sadler, E. M., et al. 2002, MNRAS, 329, 227

Sanders, D. B., \& Mirabel, I. F. 1996, ARA\&A, 34, 749

Silva, L., Granato, G. L., Bressan, A., \& Danese, L. 1998, ApJ, 509, 103

Steidel, C. C., Adelberger, K. L., Shapley, A. E., Pettini, M., Dickinson, M., \& Giavalisco, M. 2003, ApJ, 592, 728

Stocke, J. T., Morris, S. L., Weymann, R. J., \& Foltz, C. B. 1992, ApJ, 396, 487

Swinbank, A. M., Smail, I., Chapman, S. C., Blain, A. W., Ivison, R. J., \& Keel, W. C. 2004, ApJ, 617, 64

Szokoly, G. P., et al. 2004, ApJS, 155, 271

Treister, E., \& Urry, C. M. 2005, ApJ, 630, 115

Ueda, Y., Akiyama, M., Ohta, K., \& Miyaji, T. 2003, ApJ, 598, 886

Waddington, I., Windhorst, R. A., Cohen, S. H., Partridge, R. B., Spinrad, H., \& Stern, D. 1999, ApJ, 526, L77

Wang, W.-H., Cowie, L. L., \& Barger, A. J. 2006, ApJ, 647, 74

White, R. L., et al. 2000, ApJS, 126, 133

Windhorst, R. A., Fomalont, E. B., Kellermann, K. I., Partridge, R. B., Richards, E. A., Franklin, B. E., Pascarelle, S. M., \& Griffiths, R. E. 1995, Nature, 375, 471

Wirth, G. D., et al. 2004, AJ, 127, 3121

Worsley, M. A., Fabian, A. C., Barcons, X., Mateos, S., Hasinger, G., \& Brunner, H. 2004, MNRAS, 352, L28

Worsley, M. A., et al. 2005, MNRAS, 357, 1281

Yu, Q., \& Tremaine, S. 2002, MNRAS, 335, 965

Yun, M. S., Reddy, N. A., \& Condon, J. J. 2001, ApJ, 554, 803

Zezas, A. L., Georgantopoulos, I., \& Ward, M. J. 1998, MNRAS, 301,915

Zheng, W., Kriss, G. A., Telfer, R. C., Grimes, J. P., \& Davidsen, A. F. 1997, ApJ, 475, 469 
TABLE 1

Bi-lobal Source and 11 Sources Without Obvious Counterparts

\begin{tabular}{cccccccc}
\hline \hline \multicolumn{4}{c}{ Radio (J2000.0) } & \multicolumn{3}{c}{$\begin{array}{c}\text { Radio Flux } \\
(\mu \mathrm{Jy})\end{array}$} & Redshift \\
\multicolumn{3}{c}{ R.A. } \\
\hline 12 & 37 & 25.73 & 62 & 11 & 28.50 & $5960^{\mathrm{a}}$ & $1.641^{\mathrm{b}}$ \\
12 & 37 & 02.29 & 62 & 23 & 31.50 & 78.7 & $0.642^{\mathrm{c}}$ \\
12 & 35 & 40.39 & 62 & 16 & 23.80 & 60.1 & $\cdots$ \\
12 & 35 & 41.00 & 62 & 18 & 28.20 & 89.0 & $\cdots$ \\
12 & 35 & 57.71 & 62 & 08 & 08.41 & 68.6 & $\cdots$ \\
12 & 36 & 08.24 & 62 & 15 & 53.00 & $59.3^{\mathrm{a}}$ & $\cdots$ \\
12 & 36 & 24.28 & 62 & 10 & 17.00 & $54.2^{\mathrm{a}}$ & $\cdots$ \\
12 & 36 & 43.88 & 62 & 05 & 59.20 & 62.5 & $\cdots$ \\
12 & 36 & 46.70 & 62 & 12 & 26.49 & 72.0 & $\cdots$ \\
12 & 36 & 51.72 & 62 & 05 & 02.49 & $57.6^{\mathrm{a}}$ & $\cdots$ \\
12 & 36 & 54.70 & 62 & 10 & 39.60 & 48.2 & $\cdots$ \\
12 & 37 & 23.05 & 62 & 05 & 39.59 & $78.5^{\mathrm{a}}$ & $\cdots$ \\
\hline
\end{tabular}

Note.- - Units of right ascension are hours, minutes, and seconds, and units of declination are degrees, arcminutes, and arcseconds.

${ }^{a}$ Also present in the Biggs \& Ivison (2006) catalog.

${ }^{b}$ Bi-lobal source centered on a small red galaxy with optical coordinates (12:37:25.9, 62:11:29.0).

${ }^{\mathrm{c}}$ Source appears to be associated with a pair of interacting galaxies at $z=0.642$. However, because the association is uncertain, we assume this source is unidentified. 


\begin{tabular}{|c|c|c|c|c|c|c|c|}
\hline \multirow[b]{2}{*}{12} & \multirow{2}{*}{$\begin{array}{l}\text { R.A. } \\
35\end{array}$} & \multicolumn{5}{|c|}{ 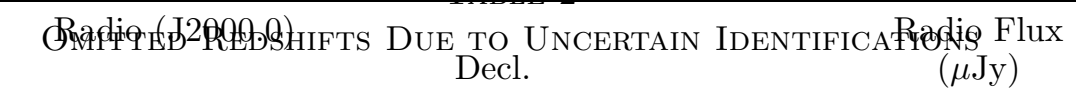 } & \multirow{2}{*}{$\begin{array}{r}\begin{array}{c}\text { Literature } \\
\text { Redshift }\end{array} \\
2.098^{\mathrm{a}}\end{array}$} \\
\hline & & 53.26 & 62 & 13 & 37.70 & 58.4 & \\
\hline 12 & 36 & 08.24 & 62 & 15 & 53.00 & 59.3 & $0.459^{\mathrm{b}}$ \\
\hline 12 & 36 & 11.41 & 62 & 21 & 49.79 & 111.0 & $0.294^{\mathrm{c}}$ \\
\hline 12 & 36 & 21.27 & 62 & 17 & 08.40 & 148.0 & $1.992^{\mathrm{a}}$ \\
\hline 12 & 36 & 36.91 & 62 & 13 & 20.41 & 50.0 & $0.680^{\mathrm{d}}$ \\
\hline 12 & 36 & 42.10 & 62 & 13 & 31.41 & 467.0 & $4.420^{\mathrm{e}}$ \\
\hline 12 & 36 & 46.70 & 62 & 12 & 26.49 & 72.0 & $2.970^{\mathrm{f}}$ \\
\hline 12 & 36 & 51.76 & 62 & 12 & 21.30 & 49.3 & $0.401^{\mathrm{g}}$ \\
\hline 12 & 36 & 56.60 & 62 & 12 & 07.60 & 46.2 & $0.321^{\mathrm{d}}$ \\
\hline 12 & 37 & 01.57 & 62 & 11 & 46.60 & 128.0 & $0.884^{\mathrm{b}}$ \\
\hline 12 & 37 & 06.77 & 62 & 07 & 22.50 & 72.8 & $0.518^{\mathrm{d}}$ \\
\hline 12 & 37 & 50.27 & 62 & 13 & 59.00 & 90.5 & $0.231^{\mathrm{d}}$ \\
\hline
\end{tabular}

Note.-Units of R.A. are hours, minutes, and seconds, and units of Decl. are degrees, arcminutes, and arcseconds.

${ }^{a}$ Chapman et al. (2005) redshift for SMM J123553.26 + 621337.7 and Swinbank et al. (2004) redshift for SMM $\mathrm{J} 123621.27+621708.4$. Each radio source has an $8 \mu \mathrm{m}$ counterpart, but there is no blue light at those positions. The redshifts appear to be of neighbor objects, which may or may not be associated with the radio sources.

${ }^{b}$ Wirth et al. (2004) redshifts for GOODS J123608.02 + 621554.0 and GOODS J123701.80+621144.2. The first radio source is far off-axis. The second radio source has an $8 \mu \mathrm{m}$ counterpart, but the redshift is of a neighbor galaxy.

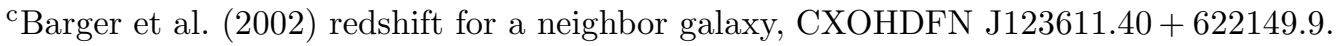

${ }^{\mathrm{d} B a r g e r}$ et al. (2000) redshifts for neighbor galaxies.

eWaddington et al. (1999) redshift. The radio source has an $8 \mu \mathrm{m}$ counterpart, but the identification is offset.

${ }^{\text {f }}$ Steidel et al. (2003) redshift. The radio source has a weak $8 \mu \mathrm{m}$ counterpart, but the redshift is of a neighbor galaxy.

${ }^{g}$ Lanzetta et al. (1996) redshift. The radio source has an $8 \mu \mathrm{m}$ counterpart, but the redshift is of a bright neighbor. 